\title{
WestVirginiaUniversity
}

THE RESEARCH REPOSITORY @ WVU

Graduate Theses, Dissertations, and Problem Reports

2013

\section{Predictors of Distress during the Breast Diagnostic Period}

Mariann M. Harding

West Virginia University

Follow this and additional works at: https://researchrepository.wvu.edu/etd

\section{Recommended Citation}

Harding, Mariann M., "Predictors of Distress during the Breast Diagnostic Period" (2013). Graduate Theses, Dissertations, and Problem Reports. 613.

https://researchrepository.wvu.edu/etd/613

This Dissertation is protected by copyright and/or related rights. It has been brought to you by the The Research Repository @ WVU with permission from the rights-holder(s). You are free to use this Dissertation in any way that is permitted by the copyright and related rights legislation that applies to your use. For other uses you must obtain permission from the rights-holder(s) directly, unless additional rights are indicated by a Creative Commons license in the record and/ or on the work itself. This Dissertation has been accepted for inclusion in WVU Graduate Theses, Dissertations, and Problem Reports collection by an authorized administrator of The Research Repository @ WVU.

For more information, please contact researchrepository@mail.wvu.edu. 
Predictors of Distress during the Breast Diagnostic Period

Mariann M. Harding

\author{
Dissertation submitted to the \\ School of Nursing \\ at West Virginia University \\ in partial fulfillment of the requirements \\ for the degree of \\ Doctor of Philosophy \\ in \\ Nursing
}

\author{
Susan McCrone, Ph.D., Chair \\ Heidi Putman-Casdorph, Ph.D. \\ Susan Coyle, Ph.D. \\ Stacey Culp, Ph.D. \\ Hannah Hazard, M.D. \\ Nan Leslie, Ph.D. \\ School of Nursing \\ Morgantown, West Virginia \\ 2013
}




\section{ABSTRACT \\ Predictors of Distress during the Breast Diagnostic Period}

\section{Mariann M. Harding}

Breast cancer is the most common cancer diagnosed in women and the leading cause of cancer deaths among women worldwide (Warner, 2011). Mortality from breast cancer in the United States has been decreasing in part due to advances in therapy and the detection of the disease at earlier stages. As a result, more than 1.6 million U.S. women will undergo a breast biopsy in 2013 with approximately 232,340 of these being diagnosed with cancer. Although distress in women undergoing a breast diagnostic evaluation has been recognized as having the potential to alter treatment outcomes in those diagnosed with cancer, few studies have explored the prevalence or predictors of distress in women undergoing diagnostic evaluations. The purpose of this study was to identify the prevalence of distress and identify predictors of distress during the breast diagnostic period. A convenience sample of 128 women (60.4\%), aged 18-89 years, undergoing core needle or surgical breast biopsy at three hospitals completed a set of nine standardized, self-report questionnaires. Distress was operationalized as anxiety and/or depressive symptoms, measured by scores on the HADS and STAI State. Varying levels of distress, manifested as symptoms of anxiety and/or depression were present with $14 \%$ of the women having symptoms above the cut-off point for clinical anxiety and $13 \%$ having symptoms of clinical depression. A clear profile emerged of factors that influenced distress and of the women who were more likely to have clinically elevated symptoms. Younger women reported more anxiety $(\mathrm{r}=-.232, p=.008)$ on the HADS-A. In multiple regression analyses, trait anxiety explained $71 \%$ of the variance on the STAI State $(\mathrm{R} 2=.842, \mathrm{~F}(1,124)=306.9, p<.001)$ and $44 \%$ of the variance on the HADS-D score $(\mathrm{R} 2=0.738, p<0.001)$. A model with trait anxiety, satisfaction with medical care, meaning in life, and friend support accounted for $66 \%$ of the variance in the HADS-D score $(\mathrm{R} 2=0.814, \mathrm{~F}(4,123)=60.4, p<0.001)$. Responses indicated that when faced with a potential cancer diagnosis, distress levels were based upon a woman's personality and her evaluation of whether she felt she had the resources to able to adapt to life with cancer. Screening protocols need to be routinely included in diagnostic radiology appointments to assess distress levels. For women with high levels of distress, interventions to decrease distress need to be tested to determine the effectiveness of providing information, facilitating communication with health care providers, and offering emotional support. 


\section{ACKNOWLEDGEMENTS}

Our lives are a journey upon a path we do not walk alone. Throughout my doctoral study, many persons have been on this path with me and I would like to express my sincerest appreciation to those who have given their time, support, and encouragement during my journey to obtaining a doctoral degree.

First to Dr. Susan McCrone, who throughout my doctoral study has been an exemplary mentor and teacher. She has been generous with offering her expertise and time, particularly through hours of class, proof reading, and completion of this study and the preceding pilot studies. Her attentive presence has helped to lay the foundation for my becoming the researcher that I aspire to be.

I would like to thank my committee members, Dr. Heidi Putman-Casdorph, Dr. Susan Coyle, Dr. Stacey Culp, Dr. Hannah Hazard, and Dr. Nan Leslie, for their time and constructive remarks. Their collective expertise has assisted this work in reaching fruition. In addition, I need to express gratitude to Dr. Mary Jane Smith, for fostering the appreciation of nursing as a science. To Margaret Rateau, Joan Lappin, and Denise McEnroe-Petitte, thank you for your encouragement and support, it is an honor to work with you and have you as friends. To my cohort, Vera Barton-Cato, Elizabeth Rini, and Crystal Sheaves, through our study your contributions have been valuable in guiding the direction of this study.

I need to offer an expression of gratitude to the faculty and staff at the West Virginia University School of Nursing, for the support offered the past five years. Finally, a special thank you goes to Lisa Cordero and the staff at the Betty Puskar Center; Leisa Arnold and the staff at Southeastern Ohio Regional Medical Center; and Marlene Hostetler and the staff at Coshocton Memorial Hospital. Without your assistance and dedicated care provided to women undergoing diagnostic evaluations, this work would not have been possible.

In closing, I dedicate this dissertation to those in my life who have been given me their unconditional love and are the most important persons in my life, my husband, Jeff Harding, my daughters, Kate and Sarah Montgomery, and my parents, Michael and Mary Mayhew. 
Table of Contents

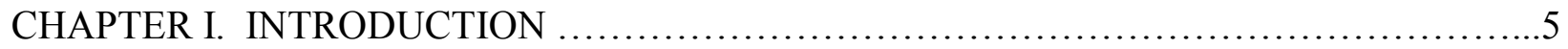

CHAPTER II. REVIEW OF LITERATURE ......................................... 15

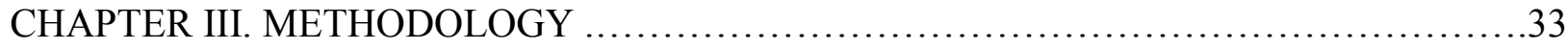

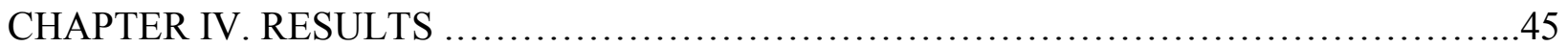

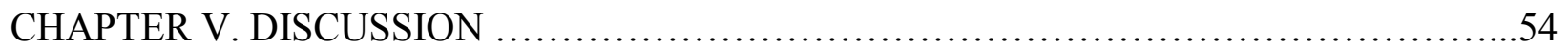

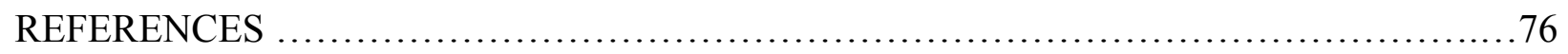

LIST OF TABLES

Table 1. Demographic Characteristics ....................................... 93

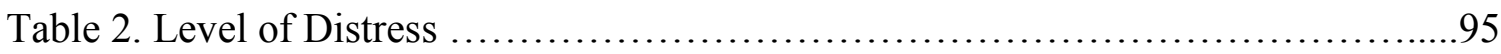

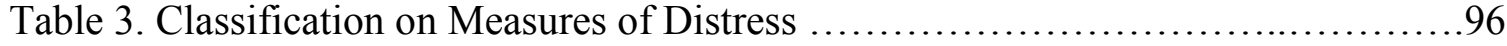

Table 4. Relationships between Distress Measures and Demographic Characteristics... 97

Table 5. Relationships between Distress Measures and Health Care ....................98

Table 6. Correlations between Distress Measures and Coping Method .................99

Table 7. Correlations between Distress Measures and Social Support.................. 100

Table 8. Correlations between Distress Measures and Personality Factors ..............101

Table 9. Differences in Distress between Young and Old Women .....................102

Table 10. Differences in Elevated Distress between Young and Old Women...........103

Table 11. Differences by Anxiety Level on STAI State Scale.......................104

Table 12. Demographic Differences by Anxiety Level on the STAI State Scale .........105

Table 13. Differences by Depression Level on the HADS-D Scale .....................107

Table 14. Demographic Differences by Depression Level on the HADS-D Scale ......109

Table 15. Multiple Regression Model for STAI State Score ........................110

Table 16. Multiple Regression Model for HADS-D Scale Score ......................111

\section{LIST OF FIGURES}

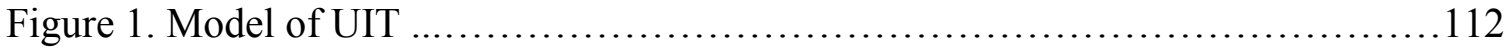




\section{Chapter 1}

\section{Introduction}

This chapter discusses the background, significance, and purpose of the study. The initial chapter provides the foundation for the significance of the research and the potential influence findings may have on nursing science, practice, and quality of life in women undergoing a breast diagnostic evaluation. Information gathered from this investigation will further the understanding of the experiences of women with suspected breast cancer and provide evidence for basing interventions aimed at managing distress associated with the diagnostic evaluation experience.

\section{Background}

Breast cancer is the most common cancer diagnosed in women and the leading cause of cancer deaths among women worldwide (Warner, 2011). Since 1990, the mortality from breast cancer in the United States has been decreasing in part due to advances in therapy and the detection of the disease at earlier stages. Advances in radiologic imaging have improved the ability to detect breast abnormalities that require further investigation to determine if a malignancy is present. As a result, more than 1.6 million women in the United States will undergo a breast biopsy in 2013 with approximately 232,340 of these being diagnosed with cancer ("Cancer Facts and Figures: 2012," 2012).

There is concern regarding the potential for prolonged adverse consequences arising from the experience of undergoing a diagnostic evaluation for suspected breast cancer (Harding \& McCrone, 2011). Discovering that there is an abnormality in one's breast is a frightening experience and most women report experiencing immediate, intense fear that they have cancer (Demir, Donmez, Ozsaker, \& Diramali, 2008; Liao, Chen, Chen, \& Chen, 2007). Uncertainty 
regarding this potential diagnosis and accompanying fear of death and the unknown has a negative effect, resulting in distress that persists throughout the diagnostic period. This distress, however, is overlooked (Brocken, Prins, Dekhuijzen, \& van der Heijden, 2012).

Being distressed can interfere with the woman's ability to obtain necessary health care, possibly leading to higher mortality in the presence of a confirmed cancer diagnosis (Allen, Shelton, Harden, \& Goldman, 2008; Ell, Vourlekis, Lee, \& Xie, 2007). High levels of distress in the diagnostic period are either sustained or increase in the immediate post diagnosis period, decreasing cognitive function, lessening satisfaction with health care, increasing postoperative discomfort, including nausea, fatigue, and pain, and lowering immune function, which increases surgical risk and potential rates of infection (Ando et al., 2011; Brocken et al., 2012; Cimprich, So, Ronis, \& Trask, 2005; Ferrante, Chen, \& Kim, 2008; G. Montgomery \& Bovbjerg, 2004; Witek-Janusek, Gabram, \& Mathews, 2007). If women are distressed, they are less able to discuss and weigh treatment options with the health care provider making them less informed and less able to actively participate in decision-making (Gilbert et al., 2011).

In women with benign disease, those with higher levels of distress may have increased apprehension regarding breast cancer resulting in behavioral changes years afterwards, affecting mammography behaviors and resulting in persistent worry regarding perceived cancer risk (Andrykowski et al., 2002; Barton et al., 2004; Brewer, Salz, \& Lillie, 2007; Lampic, Thurfjell, Bergh, \& Sjoden, 2001; Lowe, Balanda, Del Mar, \& Hawes, 1999; Olsson, Armelius, Nordahl, Lenner, \& Westman, 1999). A reduction in the likelihood of undertaking further screening could be related to anxiety stemming from the woman's desire not to undergo the experience again (Haas, Kaplan, McMillan, \& Esserman, 2001). Women overly concerned about the risk of breast carcinoma may increase utilization of self-breast examination and mammographic services 
beyond the recommended screening intervals (Aro, Pilvikki Absetz, van Elderen, van der Ploeg, \& van der Kamp, 2000). Those who experienced heightened anxiety associated with a prior experience tend to have elevated distress just prior to their next mammography exam (KeyzerDekker et al., 2012).

\section{Conceptual Framework}

For the purpose of this study, structuring uncertainty was conceptualized as drawing on inner strength to reframe the experience of disruption. A synthesis of the reviewed literature provided the foundation for developing the meanings of the four attributes of structuring uncertainty, uncertainty, disruption, inner strength, and reframing.

Mishel's (1988) middle range Theory of Uncertainty in Illness (UIT) provides the basis for the conceptual phenomenon of "structuring uncertainty" in relationship to the experiences of women undergoing breast diagnostic evaluation. Uncertainty is defined as "the inability to determine the meaning of an illness-related event, occurring when one is not able to predict the outcome accurately" (Mishel, 1988 p. 225). This disrupts everyday life by challenging the belief that one is healthy and forcing one to confront the possibility of having a potentially lifethreatening disease (Jordens, Little, Paul, \& Sayers, 2001).

The concept of disruption (Bury 1982) is widely used as a framework for understanding illness as an experience. Bury describes illness as disruptive to the structure of everyday life, life's taken for granted features, or one's life trajectory (Bury, 1982). A potential cancer diagnosis comes as a surprise and the resulting transition from health to the potential for chronic illness is a challenge to one's self. The factor that has the greatest impact on the context of perceived disruption is a woman's age. In younger women, there is a greater perception of disruption as the shift from the perceived normal trajectory to one fundamentally abnormal and 
potentially damaging is greater (Bury, 1982). Besides facing the possibility of premature death, younger women face greater concerns regarding their potential inability to perform tasks that are instrumental and dependent on physical stamina, such as working and performing family and child-rearing responsibilities (Devins, Bezjak, Mah, Loblaw, \& Gotowiec, 2006; Rasmussen \& Elverdam, 2007).

An understanding of inner strength as it encompasses well-being is necessary to facilitate the quality of life in women (Roux, Lewis, Younger, \& Dingley, 2003). While inner strength exists prior to a disruptive event, it is the existential threat that mobilizes women, making the connection with the inner self in order to meet the demands of the illness, promote health and well-being and adapt life to live as fully as possible (Baldacchino \& Draper, 2001). Dingley et al. (2000) identified personality traits related to inner strength, including: optimism, resiliency, humor, spirituality, a problem-solving attitude, and a sense of purpose or meaning in life (Dingley, Bush, \& Roux, 2000). These personality dispositions are antecedents of uncertainty, with a greater sense of these dispositions being associated with lower levels of uncertainty (Mishel, 1997).

Resilience, a dimension of inner strength comprised of the attributes of perseverance, self-efficacy, creativity, and connectedness, influences the ability to recover and achieve psychological balance after an adverse experience (Lundman et al., 2009). Women with lower resilience have more difficulty dealing with the negative effects of stress, experiencing more anxiety and depression, and do not recover as quickly when faced with adversity (Connor \& Davidson, 2003; Tugade \& Fredrickson, 2007). Women that are more resilient are more adaptable and have good coping skills (Lundman et al., 2009). Level of resilience as a 
personality trait could predict who might experience more or less distress during the breast diagnostic experience (Atkinson, 2009).

Penrod (2007) states that when there is uncertainty, one usually is distressed, prompting coping efforts aimed at managing the resulting anxiety and uneasy sensations (Penrod, 2007). Reframing is a process that guides coping efforts that assist in meeting the demands of life activities while managing uncertainty and any associated distress (Clutton, Buckley, \& Pakenham, 1999). Through this process, women reconstruct a new view of life that could accommodate a potential breast cancer diagnosis and the impact that this would have on their lives (Ching, Martinson, \& Wong, 2009).

Lazarus defines coping as constantly changing cognitive and behavioral efforts to manage specific, stressful demands within one's situational context (Lazarus, 2006). Coping strategies are classified as problem-focused coping or trying to tackle a problem actively and directly; emotion-focused coping or trying to deal with emotional reactions to problems; and avoidant coping or trying to escape from having to deal with the situation. Most contemporary models of coping suggest that when stressful situations are appraised as uncertain or uncontrollable, such as waiting for a potential cancer diagnosis, coping strategies that actively manage and handle uncertainty and distress will be most adaptive (Heckman et al., 2004). Women who rely on avoidance have the potential for dysfunctional behavior, projecting anger towards others and being overtly hostile.

\section{Theoretical Framework}

The middle-range theory of Uncertainty in Illness (UIT) provided the framework for this study. Uncertainty in illness is defined as the "inability to determine the meaning of an illnessrelated event, occurring when one is not able to predict the outcome accurately" (Mishel, 1988, p. 225). Mishel's work on uncertainty incorporates a number of principles from research 
grounded on stress and coping from Lazarus and Folkman. Lazarus states that if coping is ineffective, then stress is likely to be substantial and have damaging consequences for one's health, moral and social functioning leading to distress (Lazarus, 2006, p. 20). Mishel developed UIT to explain how one manages a specific type of stressor, uncertainty related to an illnessrelated event, and constructs meaning for that illness event.

Distress is a consequence of the experience of uncertainty (Gil et al., 2004). The level of distress depends on how one appraises and manages the illness-associated event (Mishel, 1990) Appraisal is based on experiences, personality, the interpretation concerning the severity of the illness, and the potential disruptive impact of the illness on everyday life. In this population, pertinent factors include demographic attributes, including age and the presence of children in the home; personal experiences of breast abnormalities, such as a previous history of a breast biopsy; and personality attributes, including resilience and trait anxiety.

Penrod (2007) states that when there is uncertainty, one usually is distressed responding with anxiety and uneasy sensations (Penrod, 2007). Studies of women with ovarian cancer and breast cancer support UIT by confirming that uncertainty played an important role in relation to the presence of distress, including anxiety, which women felt during the trajectory of their illness (Gil et al., 2006; Guadalupe, 2010).

Managing uncertainty prompts coping efforts directed at reducing the level of uncertainty and managing the accompanying distress. Coping through active confrontation of uncertainty and employing behaviors that one normally uses to reduce stress are the best means to attain the lowest level of distress possible. Social support functions to buffer the effects of the event through receiving information and advice; therefore, those with better social support should, theoretically, adjust better to the uncertainty experience (Mishel, 1988). Health care providers 
can directly decrease uncertainty by providing information, promoting a positive interpretation of events through reframing and facilitate coping through encouraging the use of appropriate coping methods (Mishel et al., 2002).

Mishel's UIT has been used as the framework to study the illness experience in a variety of populations, including rheumatoid arthritis, coronary artery surgery, human papillomavirus infection, diabetes, atrial fibrillation, stroke, and cancers of the breast, lung, lymph, prostate, uterus, and ovary. There is one report of the use of UIT as the framework of study for women experiencing a breast biopsy. Liao et al. (2008) used UIT to examine uncertainty and anxiety in 127 Taiwanese women during the breast diagnostic period. Uncertainty and anxiety correlated with age, marital status, educational level, religious status, family history of benign breast tumor, and the perception of the probability of receiving a breast cancer diagnosis (Liao, Chen, \& Chen, 2008). There was no examination of other key elements of UIT including social support, coping, and personality.

The manner in which Mishel conceptualizes uncertainty contributes to its suitability as the framework for the proposed study. In applying UIT to the prediction of distress, several factors should influence the existence of distress. These factors include personal characteristics, including age, a family history of breast cancer, and personality, social factors, including social support network, coping, and experiences with the health care team. If the results of this study confirm that these factors influence distress levels in a sample of women undergoing diagnostic evaluation, then the findings will lend to the generalizability of UIT, and the development of interventions aimed at mediating distress. 


\section{Significance of the Study}

The results of this study will further the body of knowledge of nursing through providing a better understanding of uncertainty as a human health experience in women undergoing the breast diagnostic experience, therefore advancing nursing science as a professional discipline. By using a middle range theory of nursing as the theoretical framework for study and examining this phenomenon through the application of this theory, knowledge will be generated that will potentially increase the generalizability of the theory to a new population.

The goal of nursing care during the biopsy evaluation period is to promote a more positive experience and achieve better outcomes through either a quicker return to a pre-biopsy emotional state or enhanced coping for those beginning the breast cancer trajectory. Nurses possess the knowledge and ability to have a direct impact on the diagnostic experience and patient outcomes. The initial step is to apply the knowledge gained from this study to the development of clinical guidelines for distress screening. The nurse, as part of the healthcare team, would be able to use these guidelines to screen women undergoing a diagnostic evaluation, quantify the level of distress, and appropriately identify those women who are in need of support that is more intensive.

Understanding factors influencing distress has the potential to lead to the implementation of specific interventions aimed at mediating distress levels. Nurses can enhance the quality of life during the evaluation period by assisting women to utilize these interventions in efforts to decrease uncertainty and lessen distress. A model of support grounded in the results of this study could delineate the various areas in which interventions are needed. This model would provide the framework for the design and testing of targeted interventions, particularly for those women at higher risk who may be in need of support that is more intensive. In women diagnosed with 
breast cancer, these interventions have the potential to alter treatment outcomes. If women are less anxious, they will be better able to discuss and weigh treatment options with the health care provider making them better informed and more able to actively participate in decision-making (Gilbert et al., 2011).

With health care costs increasing, the cost of care is an important factor in any decision regarding the provision of patient care. Absent from the literature is any discussion of the relative benefits in relationship to cost of providing women support during the diagnostic period. Since budgetary restrictions could be a potential issue in providing services to women during this period, the results of this study could provide evidence to support policy decisions regarding funding for case management or navigation programs aimed at providing women support during the diagnostic period. This would be particularly beneficial to those who are experiencing distress, particularly at higher levels, who are in need of assistance with navigating the health care system, or who need practical assistance with finances, transportation, or childcare.

\section{Purpose}

The purpose of this study was to identify the prevalence of distress and evaluate predictors of distress during the breast diagnostic evaluation period. This study was innovative in that the aim was perform a comprehensive evaluation of factors that influence distress and develop a profile of women who may be at higher risk for elevated levels of distress associated with the breast diagnostic process. Applying a new understanding of factors influencing distress could lead to the implementation of screening protocols and interventions that have the potential to improve the quality of women's care during the diagnostic experience and alter treatment outcomes and quality of life in those diagnosed with breast cancer. 


\section{Research Questions}

This study examined the following research questions:

1. What is the prevalence of distress present in women during the breast diagnostic experience?

2. Are there significant relationships among distress and demographic characteristics, satisfaction with medical care, coping method, social support, and personality factors?

3. Are there significant differences between younger and older aged women in the level of distress experienced?

4. Are there significant differences between women with and without elevated distress and demographic characteristics, satisfaction with medical care, coping method, social support, trait anxiety, resilience, and meaning of life?

5. What are the predictors of distress in women undergoing a breast diagnostic evaluation for suspected cancer? 


\section{Chapter 2}

\section{Review of the Literature}

This chapter will provide an overview of the literature search process and a synthesis of current research on the topic of women's experiences during the breast diagnostic evaluation period. This synthesis includes conceptual, methodological, and empirical knowledge from quantitative and qualitative studies. The chapter will conclude with a description of Uncertainty in Illness theory and a discussion of how quantitative methodology was appropriate to use with this theory as the framework for study.

\section{Literature Search Process}

A systematic literature search was conducted to investigate the experiences of women undergoing a breast diagnostic evaluation and provide support for the proposed research study. The search was conducted in the CINAHL, MEDLINE, Dissertation Abstracts and PsycINFO databases for studies published in the English language between January 1983 and January 2012 using search terms in the title, abstract, or keywords, then ancestry and descendancy approaches. Dissertation Abstracts was included to minimize possible bias towards published studies. The use of a broad timeframe ensured identification of the vast majority of published studies using the search terms. Search terms were breast cancer diagnosis, mammography, breast biopsy, breast diagnostic, anxiety, distress, and uncertainty. Each diagnostic related term was entered into the keyword function, then combined using the AND function with the psychological terms.

Examination of identified studies started with an appraisal of the titles and abstracts to determine if they met inclusion criteria. The following were the inclusion criteria:

1. Described an aspect of a woman's experiences during the breast diagnostic evaluation period defined as beginning when a woman becomes aware of a confirmed mammographic abnormality 
and ending when the woman receives notification of the results of diagnostic procedures that confirm or rule-out whether a malignancy exists

2. Quantitative studies utilized a design that included at least one variable that was a standardized or validated measure of distress

3. The study presented new information not already reported in an earlier source The following were the exclusion criteria:

1. The purpose of the study was instrument or program development.

2. The study examined mammography-screening behaviors

If a study met the inclusion criteria, a critical appraisal of the full text occurred. Information extracted from each study included the author, country of origin, year published, purpose of study, design, sample size, data collection method, variables, measures, analytical methods, rigor, and major findings.

\section{Literature Review}

\section{Methodological}

The review process identified 36 quantitative and 7 qualitative studies. Several methodological limitations affect the generalizability of findings reported in the current research literature and guide the direction of the proposed study. The majority of studies originated outside of the United States, which accounted for only 18 studies. Other studies were conducted in Canada (seven), the UK (four), Taiwan (three), Australia, Japan, and Sweden (two each), and Iceland, Ireland, Israel, Norway, and Turkey (one each). Since actions in everyday life reflect cultural values, including how one copes, uses social support, discloses information, and makes decisions, there is the potential for a lack of generalizability of findings from these other studies to the overall experience of women in the United States. All eight of the multisite studies were conducted outside of the United States. 
Most of the quantitative studies were multidisciplinary and were composed of teams of psychologists, physicians and nurses. Only fourteen quantitative studies cited a nurse as first author; in contrast, a nurse performed or was first author of every qualitative study. The majority of the studies did not report the use of any theoretical framework. When one was cited, the most commonly used was Lazarus and Folkman's Transaction Model of Stress and Coping (5 studies). Two others utilized other coping theories as the framework for study. Only one study used a nursing theory as the theoretical framework of study; the nursing theory used in that instance was Uncertainty in Illness Theory.

The characteristics of the women included in the samples varied widely. In the studies from 1983-1993, all of the women had a biopsy as a hospital inpatient, reflecting the standard of care at that time. Practice has evolved to include two primary biopsy techniques, surgical, including excision and lumpectomy, and the current standard, core needle with imaging guidance. A number of studies used type of biopsy as an inclusion criterion, with several only including women undergoing surgical biopsy. Some studies initially included all women who were undergoing a biopsy, only to later exclude data from those diagnosed with breast cancer, or only sampled those undergoing an excisional biopsy. Many chose to exclude women with a previous history of breast biopsy or a personal history of breast or other cancer. A few only included those with abnormalities identified on screening mammography; excluding those with self-discovered abnormalities or accompanying symptoms. Several limited the ages of women, including only ages 50-69, over 40, or over 50 .

There were a few issues with statistical analyses. Sample sizes ranged from 25 to 623 . Nine studies had sample sizes that were lacking sufficient statistical power to detect true differences among subgroups. Only four studies included a priori power analysis. While 
reported analyses matched the type of data collected, often there was appropriate data that could have been used to perform advanced statistical tests that would have strengthened study findings. Eight studies only used correlational statistics or tested for group differences, when data to conduct regression was available. Other scenarios included a mix of performing independent $\mathrm{t}$ tests or regression and neglecting to test group variances.

\section{Measurement of Distress}

There has been no comprehensive evaluation of factors that influence distress. Many studies focused on examining the relationship between two primary sets of correlates, such as distress and social support or coping, coping and social support, or distress and demographic factors. In addition, too few studies have examined some of the correlates that may influence distress to allow any firm conclusions to be drawn.

The absence of specific instruments to measure distress and clear conceptual and operational definitions for distress, has led to the use of many different instruments to measure distress (Harding \& McCrone, 2011). Distress has been assessed using well-validated, quantitative tools that measure a variety of concepts, including depression, numbness, compliant tendencies, arousal, optimism, hostility, paranoia, hopelessness, worry, anger, tension, intrusion, moodiness, and anxiety. Variations of the State-Trait Anxiety Inventory (STAI) scale have been the most widely used instrument to measure anxiety in women undergoing breast biopsy as well as with women who had breast cancer (Harding \& McCrone, 2011).

Researchers have used two other instruments to measure anxiety in this population. The Psychological Consequences of Screening Mammography (PCQ) and the Breast Cancer Anxiety Indicator (BCAI) have been reported to strongly correlate with anxiety levels during the period between an abnormal mammogram and during the wait for additional test results (Pineault, 
2007). However, the PCQ is intended to measure the positive and negative effects related to participating in screening mammography, which is not equivocal to undergoing a breast biopsy(Brodersen, Thorsen, \& Kreiner, 2007). The BCAI is a single item question, "how do you rate your anxiety in relation to breast cancer during the last week," and was intended to measure anxiety related to thoughts regarding potential cancer during the screening mammography process (Meystre-Agustoni, Paccaud, Jeannin, \& Dubois-Arber, 2001).

Coping. There are many ways to conceptualize coping strategies and the use of a several different measures to examine coping strategies reflects the variations in conceptualization. The most frequently used measures of coping were variations of either the Cope Inventory (COPE), or the Coping and Defense Inventory (CODE), which consists of the Utrecht Coping List and the Defense Mechanism Inventory (Harding \& McCrone, 2011). The COPE measures how often respondents use certain strategies to cope with stress. Subscales of the COPE relevant to this population include self-distraction, positive reframing, emotional support, humor, planning, denial, religion, and active coping (Lebel et al., 2003).

Social support. Similarly, in efforts to examine the relationship between social support and the experience of undergoing a breast biopsy, researchers defined and examined social support in various ways across studies. These included very narrow definitions of social support defined only in terms of the number of persons in a woman's support network to more broad conceptualizations of support as the global perception of the quality of emotional and instrumental support. Some instruments evaluate perceived support from the entire network, not allowing respondents to separate out the type of support received from a spouse from the support received from a friend. 
Qualitative methodology. Qualitative studies have provided rich information through insights into the lived experiences of woman undergoing breast evaluations. Most of the qualitative studies used a phenomenological approach with unstructured or semi-structured interviews. The study that was the exception used a series of focus groups for data collection. Each interview and focus group session was audio taped and transcribed verbatim. Researchers analyzed the texts for overall patterns and identified conceptual themes within the data to gain understanding and meaning of the women's stories. Most studies reported coding data in two stages; an initial coding to identify categories followed by a more focused coding to develop the themes that emerged from the stories. To establish validity and reliability, at least two people in addition to the primary researcher reviewed copies of each set of transcripts and data analyses for credibility.

\section{Empirical}

Distress. The uncertainty experience begins the moment of discovery. Most women report experiencing immediate, intense fear (Demir et al., 2008; Liao et al., 2007); other initial reactions include feelings of injustice, particularly if there were no perceived risk factors, and disbelief (Chappy, 2004). Uncertainty surrounding the potential diagnosis is disruptive, resulting in distress that persists throughout the diagnostic period. Anxiety appears to be the most specific manifestation that characterizes this distress (Harding \& McCrone, 2011).

Researchers have consistently been able to document the presence of anxiety throughout the diagnostic period. This is not surprising. Within the context of UIT, the uncertainty regarding the potential outcome is appraised as a threatening, resulting in distress and the accompanying sensation of anxiety. In comparison, when the existence of distress during the diagnostic period is defined through the use of concepts such as worry and depression the 
prevalence of distress is low (Andrykowski et al., 2002; Harcourt, Rumsey, \& Ambler, 1999;

Lampic et al., 2001; G. H. Montgomery et al., 2003; Potter, 2007; Schnur et al., 2008).

Women however experience different degrees of distress. In small cohorts of women, some researchers have been able to define levels of anxiety that were above the cut-off point for clinically defined anxiety disorder (Harding \& McCrone, 2011). The level of anxiety can interfere with critical thinking and information-processing abilities (DeKeyser, Wainstock, Rose, Converse, \& Dooley, 1998; Liao et al., 2008; Poole et al., 1999). There appears to be a correlative relationship between trait and perceived levels of anxiety (de Vries, van der Steeg, \& Roukema, 2009; Iwamitsu et al., 2005; Maxwell et al., 2000; Novy, Price, Huynh, \& Schuetz, 2001); the chronically anxious tended to have more anxiety before biopsy and after diagnosis. In regression models, the strongest predictor of anxiety levels is trait anxiety (Ando et al., 2009; Chen et al., 1996; Iwamitsu et al., 2005; Maxwell et al., 2000; Novy et al., 2001).

One published interventional study aimed to decrease anxiety. Potter (2007) offered two REIKI interventions to 16 women scheduled for a breast biopsy. While the women in the intervention group reported that they felt better, there was no difference or decline in anxiety levels between the intervention and control groups (Potter, 2007). No other research has addressed current practices concerning whether the presence of distress was routinely screened outside of study protocols or the effectiveness of any interventions that were already in place to assist women in alleviating psychological distress levels (Harding \& McCrone, 2011; Mertz et al., 2012). This leads to the conclusion that distress may not be formally recognized or adequately treated by healthcare teams.

Four studies assessed the relationship between level of psychological distress and immune factors that may affect operative outcomes. Witek-Janusek (2007) found reduced 
natural killer cell activity and interferon production and increased production of certain interleukins and stress hormones before biopsy that continued for four months afterwards. These results support earlier findings from testing performed just one week following biopsy (Birney, 1995; Walter, 2005). DeKeyser et al. (1998) found statistically significantly altered levels of the tumor necrosis factor that correlated with psychological distress only in those diagnosed with malignancy; however distress in this instance was inferred from measures of symptom distress, as opposed to using a validated measure of anxiety. Although the biochemical measures of immune factors appear to have potential as psychological distress markers, these studies all had small sample sizes and extensive lists of exclusion criteria including concurrent immune-based disease or using an immune function-altering medication.

\section{Factors Influencing Distress}

Demographic Characteristics. There has been some examination of the relationships between distress levels and demographic variables, particularly medical history, age, and education. Since women with a history of certain benign breast diseases have an increased risk for breast cancer, it is not surprising that those women who have undergone previous breast biopsy report higher levels of anxiety (Deane \& Degner, 1998; Haas et al., 2001; Lebel et al., 2003; Liao et al., 2007). Compounding this heightened anxiety are previously negative experiences (Lampic et al., 2001; Pineault, 2007). A personal history of cancer, a family history of cancer and the presence of co-existing diseases correlate with increased distress (Andrykowski et al., 2002; Lebel et al., 2003; Schnur et al., 2008).

The weight of the evidence regarding age is inconclusive. While some studies have found that age may not have any influence on distress (Drageset \& Lindstrom, 2005; Northouse, Jeffs, Cracchiolo-Caraway, Lampman, \& Dorris, 1995; Olsson et al., 1999), others contradict 
these reports stating that younger women experience increased distress (Chen et al., 1996; Haas et al., 2001; MacFarlane \& Sony, 1992; Seckel \& Birney, 1996; Stanton \& Snider, 1993). The fact that there is no substantiation of an association between younger age and increased distress is surprising. It is well documented that younger women with breast cancer, as well as those with ovarian and gastrointestinal cancers, have demonstrated increased distress (Anderson, Ganz, Bower, \& Stanton, 2012; Arden-Close, 2008; Cesario, Nelson, Broxson, \& Cesario, 2010; Giske \& Artinian, 2008; Mertz et al., 2012). At a younger age, a potential cancer diagnosis is more likely to affect everyday life. There would be a number of key issues including the impact of a diagnosis on one's spouse and children, the possibility of an early death and unfilled goals, and the loss of femininity and sexuality associated with surgical procedures. Anecdotal notes in the quantitative literature provide some description of the concern women with younger children had for the children's future and the effects a cancer diagnosis would have on them. Because some evidence points to an association between younger age and more distress, there is a need for additional research regarding the relationship of age and distress. Definitive findings confirming the presence of increased distress would affirm the health care team's need and responsibility to provide younger women with adequate support.

Coping Mechanisms. Research has indicated that women engage in a wide range of coping behaviors during the diagnostic period. The use of specific coping strategies has been found to influence distress levels during the diagnostic period (Chen et al., 1996; Heckman et al., 2004; Lebel et al., 2003) and be indicators of psychological adjustment after surgery (Degner, Hack, O'Neil, \& Kristjanson, 2003; Drageset, Lindstrom, \& Underlid, 2010). The most helpful coping strategies are either active, in which the women perform activities to assist them to dealing with the problem, or emotion focused, in which the women try to deal with the distress 
they are experiencing. Other strategies that have been reported are participating in activities that have assisted with reducing stress previously, such as listening to music or exercising, or participating in alternative activities that are considered treats, such as going shopping, or special outings (Logan, Hackbusch-Pinto, \& De Grasse, 2006). Some women use behavioral avoidance, denying the experience is occurring, or participate in alternative activities, such as smoking, sleeping, antianxiety medication use or drinking more alcohol more often (Heckman et al., 2004). Using avoidance, aimed at distancing oneself from the stressor, is associated with the highest levels of distress (Drageset \& Lindstrom, 2003; Harcourt et al., 1999; Lebel et al., 2003; Stanton \& Snider, 1993).

Attributes of Inner Strength. Outside of evaluating optimism, there has been no exploration of the role of the attributes of inner strength on distress, despite the fact that these attributes may influence distress levels. Higher levels of optimism may mediate distress directly by contributing to expectations of a positive outcome (Lauver \& Tak, 1995; Northouse et al., 1995), but lower levels of optimism have not been found to be associated with higher distress (G. H. Montgomery et al., 2003). It is plausible that optimism plays a significant role in helping women cope by influencing the selection of coping strategies and helping them to have higher expectation of the quality of care that they will be receive (Andrykowski et al., 2002; Lauver \& Tak, 1995; Logan et al., 2006). A comparison of women who reported low levels of anxiety throughout the diagnostic period, despite reporting the use of a variety of different coping strategies, suggests that personality type, and not the selection of coping strategies or demographic factors, may be the contributing factor in their apparent composure (Poole et al., 1999). Given the dearth of study in this area, there is a need to examine the roles of inner strength attributes, such as life meaning or resilience, in relation to uncertainty and the 
diagnostic process, especially given that a greater sense of these dispositions should be associated with lower levels of uncertainty (Mishel, 1997).

Health Care. Interactions with the health care team are highly influential on the experience of undergoing a breast evaluation. Simply put, it is necessary for women to have information regarding their risk of breast cancer and the tests to diagnose and treat the disease. Women adequately informed about the process experience less distress, cope better with the possibility of having cancer, participate actively in decision-making and have a greater degree of trust in the healthcare team (Demir et al., 2008; Drageset \& Lindstrom, 2005; Liao, Chen, Chen, \& Chen, 2010). Having a specific professional for women to contact with questions and to clarify any information lessens distress as does receiving explanations regarding diagnostic results as soon as possible (Bradley, Berry, Lang, \& Myers, 2006; Liao et al., 2007; O'Mahony, 2001).

Unfortunately, most women report feeling they received inadequate information or preparation for diagnostic procedures (O'Mahony, 2001). Most women reported only receiving verbal information, and many reported that they did not remember all of what they were told nor were given any written materials (O'Mahony, 2001). This insufficiency has several consequences. First, women spend time actively seeking outside information to fulfill this need (Allen et al., 2008); and they report finding it difficult to access the information they require (Robinson-White, Conroy, Slavish, \& Rosenzweig, 2010). In hindsight, they often feel that they were inadequately prepared for what was later described as painful, gruesome procedures (Thorne, Harris, Hislop, \& Vestrup, 1999). A lack of information also drives women to attempt to interpret the information they do possess for cues, including estimating their risk of having a malignancy, evaluating the physician's description of the suspicious abnormality or inferring 
about the speed of referral times (Poole \& Lyne, 2000). Their interpretations may lead to invalid perceptions of their situation with the majority over-estimating their risk of breast malignancy (Lebel et al., 2003).

The woman's educational level may play a role in the management of information. Women with a lower level of education may experience distress due to a lack of access to information, not fully understanding the information they were given and having fewer social resources (Andrykowski et al., 2002; Northouse et al., 1995; Novy et al., 2001; Olsson et al., 1999; Rehnberg, Absetz, \& Aro, 2001). Women with a higher level of education are at risk for experiencing greater distress related to a disparity between the quality and amount of information desired and dissatisfaction with the amount of information received (Deane \& Degner, 1998; Liao et al., 2007; Rehnberg et al., 2001).

No study has examined women's preferences or sources of additional information to fill their perceived knowledge gaps. Family, friends, television, and print media are potential sources. However, with the increased access to the Internet and the vast amount of information available, no study has queried women regarding their use of the Internet as a source of desired information. The possibility exists that using the Internet as an information source may alleviate some distress by helping women feel more prepared.

The general attitude of health care providers is equally influential. Women needed to feel that they were being treated with respect and care during the diagnostic process (O'Mahony, 2001). They desired compassion and support in their interactions with health care providers. While a positive attitude was important, assurances of "don't worry" were not comforting, but appeared patronizing to the feelings that the women were experiencing. A number of women expressed that they felt vulnerable, and at times dehumanized by the diagnostic process (De 
Grasse, Hugo, \& Plotnikoff, 1997; O'Mahony, 2001). Women stated feeling that the health care team did not treat them as an individual or recognize the personal significance of their experience (Northouse, Tocco, \& West, 1997).

There are considerable differences in duration of the diagnostic period, ranging from one day upwards to 12 weeks. It would be intuitive that receiving a speedier diagnosis from a 'onestop' clinic or diagnostic interval of less than one week would lessen distress. There is evidence to the contrary. Waiting simply sustains a given level of distress, and shortening the time to diagnosis primarily improves patient satisfaction (Hislop et al., 2002; Lebel et al., 2003; Poole et al., 1999; Schnur et al., 2008). Surprisingly, in those diagnosed with malignancy, rapid communication of biopsy results may have been detrimental. Those diagnosed with breast cancer through a one-stop clinic system had significantly higher levels of depression and anxiety during the first three months of the breast cancer trajectory (Dey et al., 2002; Harcourt et al., 1999; Shapiro, McCue, Heyman, Dey, \& Haller, 2010). This suggests that a speedier diagnosis of a malignancy may have a detrimental impact. Not having the time during the diagnostic interval to begin to come to terms with the potential diagnosis and visualize what a life with breast cancer may be like could trigger an acute stress syndrome (Harcourt et al., 1999; Lampic et al., 2001; Poole et al., 1999).

There are a few studies examining the impact of health care interventions on the diagnostic experience. Ong and Austoker's (1997) retrospective analysis found that women at English breast screening centers where a nurse provided counseling regarding diagnostic mammography findings had lower distress levels $(p<.001)$ and increased satisfaction with information received from the health care team $(p<.001)$ (Ong \& Austoker, 1997). In contrast, Hislop's (2002) secondary analysis of Canadian women enrolled in a pilot program aimed at 
reducing the diagnostic interval found that counseling provided by nurses did not have any effect on distress nor was there any difference in satisfaction with the information received by the intervention and control groups (Hislop et al., 2002). However, they provided no description of the type of counseling or educational support provided by the nurse.

Two studies focused on navigation, particularly expediting the diagnostic process. Barton (2004) performed a controlled trial to compare the effects of both the immediate reading of mammograms and of an educational intervention on the psychological status of women receiving a screening mammogram. The educational intervention consisted of a videotape and an educational pamphlet designed to reduce anxiety by providing explanations of mammography, follow-up procedures and coping tips focusing on avoidance techniques. Women received no intervention, the immediate reading of the mammogram or the educational intervention, or both interventions. Three months following the screening mammogram, researchers sampled 1037 women who had an abnormal screening mammogram without a diagnosis of breast cancer. Though women commented positively on the educational intervention, stating that it was helpful (70\%), there were no differences in anxiety levels between the educational intervention and control groups (Barton et al., 2004). One possible explanation for this disparity is that while the educational intervention provided information on diagnostic procedures, the stress management advice encouraged women to use avoidance techniques to cope with any distress they experienced. Using avoidance techniques, as discussed earlier, is associated with the highest levels of distress (Drageset \& Lindstrom, 2003; Harcourt et al., 1999; Lebel et al., 2003).

Ferrante et al. (2008) examined the effectiveness of a patient navigator on decreasing anxiety and increasing satisfaction by guiding women through the health care system to help 
ensure a timely diagnosis (Ferrante et al., 2008). Although more women in the intervention group were diagnosed with cancer, anxiety levels after diagnosis were significantly lower $(p<.001)$ and satisfaction with care higher $(p<.001)$ than for women in the control group. Ferrante et al. attributed their findings solely to the differences in time-to-diagnosis between groups (42 versus 25 days) (p. 121). Given that the patient navigator focused on the specific needs of women by providing emotional support, education, and facilitating communication with healthcare providers, it is possible, given that other studies support the role of these factors in mediating distress, that the findings were the result of the patient navigator's role rather than time.

Only one study has evaluated the effectiveness of an intervention on distress specifically during the diagnostic evaluation period. Liao et al. (2010) used a quasi-experimental design to investigate the effects of a supportive care program for Taiwanese women undergoing breast biopsy. The experimental group $(n=62)$ received a supportive care program that included a set of three purposely written education pamphlets about breast cancer diagnosis and treatment, three face-to-face sessions of information and emotional support, and two follow-up telephone consultations. The control group $(n=60)$ received routine care which included information and emotional support and referral services. The anxiety levels of women diagnosed with cancer who received supportive care were significantly lower than those receiving routine care before biopsy and after diagnosis $(p<.001)$. However, in those who received a benign diagnosis, there were no significant differences in anxiety level between the groups before biopsy, only after diagnosis (Liao et al., 2010).

Social Support. The relationship between social support and distress is complicated. There is only moderate quantitative evidence that better perceived social support is associated 
with decreased levels of distress. Yet when asked to describe the role support plays during the evaluation period, women place an extremely high value on social support (Allen et al., 2008; Fridfinnsdottir, 1997). During this time, women need and seek continuous emotional support and comfort from those in their support network. This network is the subset of persons in the woman's life on whom she relies for socio-emotional or physical aid, including husband, partner, family, friends, and colleagues (O'Mahony, 2001). There may also be benefit in connecting with women who have previously undergone a breast diagnostic procedure. These "related others" were able to provide specific information about the experience that the women were not able to find elsewhere (Thorne et al., 1999).

Most women expect their husbands or significant others to provide support. However, many women felt that the level of support they anticipated others to provide never happened (Fridfinnsdottir, 1997). Few studies have explored the capability of husbands or significant others to provide support. Shaw et al. (1994) reported that significant others often felt left out and they did not receive the information that they needed (Shaw, Wilson, \& O'Brien, 1994). This coincides with reports that husbands have similar levels of anxiety as their wives during this period (Northouse et al., 1995; Northouse et al., 1997). Moreover, while both parties wanted the husband or significant other to be present as part of the information process described earlier, the husband or significant other was often not included in information interactions with the health care team (De Grasse et al., 1997; Northouse et al., 1997). This supports findings that married women have different needs for information from their physicians. This has been attributed to having to convey information to husbands who were not present during physician visits (Liao et al., 2007). 
While a high value is placed on social support, it is uncertain how using social support is comforting or if it directly contributes towards alleviating distress. It would be helpful to know how women use their social resources and if increasing perceptions of support diminishes distress. It may be that the most effective person to be the primary source of support is not the husband or significant other, particularly if these people need to manage their own anxiety or are experiencing their own difficulties in dealing with the emotional aspect of the experience.

Living the Wait. Qualitative investigations have provided rich insight into the lived experiences of undergoing a diagnostic evaluation. Each woman, no matter how long it had been since undergoing a diagnostic evaluation, seems to have no difficulty remembering the minute details of her evaluation experience. For many women, the evaluation period has been described as a limbo period in their lives in which their priorities were altered, and they were just focused on getting through their usual daily activities and maintaining routines (Heckman et al., 2004; Shaw et al., 1994; Thorne et al., 1999). Many women experienced disruptions in their routines, including insomnia, panic attacks, and an inability to concentrate (Thorne et al., 1999).

Given the potential gravity of the situation, women often felt a need to engage in a period of isolation, focusing on themselves and their families, and in reflection, searching for meaning in the experience (Chappy, 2004). They describe undergoing 'preparatory' psychological processes, rehearsing what life with breast cancer would be like or imagining what they would do if their life span was to be shortened to only five more years (O'Mahony, 2001; Poole et al., 1999). This reflection may assist with coping with further disruption and reflect a reliance on inner strength.

Many women reported relying on their spirituality to help them handle the experience and described how their faith helped them cope. Faith allowed them to place their trust in God 
and to come to acceptance with whatever path God had chosen for them (Chappy, 2004; Logan et al., 2006; Shaw et al., 1994). Prayer was ongoing; women typically prayed for a benign outcome, that the lump would turn out not to be cancer, and for help and guidance for whatever was in store (Demir et al., 2008; Riese, 2001). For some, the experience triggered an increased awareness of spirituality, resulting in returning to church after a period of absence or seeking extraordinary prayer intentions.

\section{Summary}

The preceding literature review provided an overview of the literature search process and a synthesis of current research on the topic of women's experiences during the breast diagnostic evaluation period. Although studies confirmed some of the needs of women during this time, there has not been enough examination regarding the predictors of distress during the evaluation period to draw firm conclusions. Therefore, there is a need to further the understanding of the predictors of distress in women undergoing diagnostic evaluation to begin positively influencing women's experiences. 


\section{Chapter 3}

\section{Methodology}

This chapter provides an overview describing the methodology selected for data collection and analysis for determining the prevalence of distress and evaluating predictors of distress in women undergoing breast diagnostic evaluation. This outline will cover key definitions, and describe the recruitment of participants, human rights protection measures, data collection procedures, data analysis, and maintenance of rigor.

\section{Design}

\section{Description}

This study used a cross-sectional survey design to determine predictors of distress in a convenience sample of women undergoing a breast biopsy. Quantitative methodology is congruent with the proposed research questions since the aim is to determine the prevalence of distress and evaluate predictors of distress in women undergoing breast diagnostic examination. Given the nature of the phenomenon of interest, the use of a cross-sectional design was appropriate for capturing the experiences of women at a specific point in the diagnostic evaluation period.

\section{Definitions}

1. Breast diagnostic evaluation period. The diagnostic evaluation period begins when a woman becomes aware of a breast abnormality and ends when the woman receives notification of the results of diagnostic procedures that provide a confirmed diagnosis.

2. Distress. Distress is defined as a state of unpleasant emotions of a psychological, social and/or spiritual nature extending along a continuum, ranging from common normal feelings of vulnerability and fear to psychological states that can become disabling, including 
depression and anxiety (Howell, 2010). Therefore, total and subscale scores on two instruments that detect the presence of anxiety and/or depression, the Hospital Anxiety and Depression Scale (HADS) and the STAI, were used to measure distress as an outcome.

3. Age. Consistent with published research, women under age 50 were categorized as young; women over the age of 50 were categorized as old (Harding \& McCrone, 2011).

\section{Facilities}

This was a multisite study, utilizing three outpatient radiology clinics: Two community hospitals in eastern Ohio and one major medical center in West Virginia.

The Betty Puskar Breast Care Center, part of the Mary Babb Randolph Cancer Center at West Virginia University, is designated a National Accreditation Program for Breast Cancer Center. As part of this accreditation, the center must provide a multidisciplinary approach to coordinate the best care and treatment options available, access to breast cancer-related information, education, and support, ongoing monitoring, and screening to identify those with distress ("NAPBC Accreditation," 2012). Therefore, it may be expected that women undergoing breast diagnostic evaluations may receive more sophisticated care at this site in comparison to the non-accredited community hospitals, impacting satisfaction with care (Winchester, 2008).

Two community hospitals, Coshocton County Memorial Hospital, Coshocton, Ohio, and Southeast Ohio Regional Medical Center, Cambridge, Ohio, provide a lower volume of services to breast care patients. Neither facility delivers comprehensive treatment by a multidisciplinary team to breast cancer patients.

\section{Protection of Human Subjects}

Before data collection began, the institutional review board approved the study. During the enrollment process, the investigator or co- investigator informed potential subjects of the 
purpose and procedures of the study. The investigator or co- investigator assured each women of the confidential treatment of personal information and described the procedures used to assure this confidentiality. The investigator or co- investigator reminded each woman that her participation in the study was voluntary and that she was free to withdraw at any time without affecting her right to treatment. The investigator welcomed inquiries from the subjects, along with remarks and/or observations concerning the study.

There was no direct medical benefit to participants. Subjects may have obtained additional indirect benefit from their participation, including the opportunity to feel useful and helpful to others. Participation in this study exposed the subject to minimal risks, physical, psychological, and/or social. However, the content of the information disclosed by one or more participants may have created anxiety, fear, discomfort, and/or psychological distress. If a woman had experienced distress from study participation, the investigator would have directed the woman to counseling through local support services; no one contacted the investigatorr to initiate support services.

The only potential risk was the violation of confidentiality. To assure privacy, specific measures were undertaken. Each set of completed instruments was marked with an assigned code number. Only the code number appears on the computer data files and data collection records. The database is accessible only through passwords assigned by the investigator. The investigator secured completed instruments in a locked cabinet in the locked office of the investigator. Data and instruments will be stored for three years following the completion of the study, after which time the investigator will erase computer information, then shred and burn the completed instruments. The reported findings include only aggregate data. 


\section{Sample Selection}

\section{Description}

The study utilized a convenience sample, using self-selection, non-random and nonprobabilistic sampling. In attempts to reduce potential bias, subjects were recruited without regard to demographic characteristics.

\section{Criteria}

\section{Criteria for inclusion}

(i) Each subject presented with a suspicious mammogram and underwent a core needle biopsy or surgical biopsy as determined by physical examination and diagnostic imaging.

(ii) Female, aged 18 years or older

(iii) Able to read English

\section{Criteria for exclusion}

(i) Pregnant women. The minimal research with pregnant women with a potential breast cancer diagnosis suggests their experience may be significantly different.

(ii) Males. Research suggests that their experience is significantly different from women.

\section{Data Collection}

\section{Procedure}

The investigator or co- investigator identified potential subjects from the list of patients scheduled in the radiology department at each site to determine who met inclusion criteria. The investigator or co- investigator approached each potential subject while she was in a private room and presented her with a cover letter describing the purpose of the study, the risks, benefits, and an affirmation of the right to withdraw at any time from the study. Within the letter, the 
investigator clearly identified self, affiliations, and provided contact information so that potential subjects could verify the legitimacy of the study. Those agreeing to participate received a packet containing the instruments and a prepaid postage return envelope. Each packet had a simple designated code on the return envelope representing each site.

The woman completed the instruments at the facility or at home. If the woman elected to complete the instruments at home, she was asked to return them to the investigator within a week, before receiving a final diagnosis. If a woman elected to complete the questionnaires at the facility, she was allowed to remain in the private area while doing so. After completing the instruments, the woman placed them in a sealed envelope and gave the envelope to the investigator or co- investigator. The investigator retrieved the questionnaires unopened from the co- investigator. Data analysis began as soon as the investigator received completed instruments.

\section{Variables}

Data was collected using self-report questionnaires. This set of empirically supported instruments was chosen based on the conceptual and operational definitions of key variables and for their potential efficacy in the evaluating correlates of distress in women undergoing breast diagnostic evaluation. The following questionnaires were administered in this order:

1. Demographic questionnaire. Using a purposefully devised tool, demographic characteristics collected included age, level of education, employment status, presence of children under the age of 18 in the home, relationship status, family history of breast cancer, personal history of any cancer, and history of a prior abnormal mammogram or breast biopsy. Each factor was a separate independent variable.

2. PSQ-18. The Patient Satisfaction Questionnaire has seven different subscales: general satisfaction, technical quality, interpersonal manner, communication, financial aspects, 
time spent with doctor, accessibility, and convenience. The PSQ-18 is a selfadministered questionnaire with responses rated on a 5-point Likert scale ranging from 1, strongly agree, to 5, strongly disagree. Items are the PSQ-18 items are worded and scored so that higher scores reflect greater satisfaction with medical care. Reliability coefficients for the subscales are reported to range from .64 (communication) to .77 (time spent) (Marshall \& Hayes, 1994). The Cronbach's coefficient in this study for the total PSQ-18 was .95. The total score on the PSQ-18 was used as an independent variable.

3. HADS. The Hospital Anxiety and Depression Scale measured distress. It consists of 14 multiple-choice questions within two subscales, seven questions measuring anxiety (HADS-A) and seven depression (HADS-D). The depression sub-scale evaluates the degree of anhedonia, the loss of pleasure or interest in life, lack of enthusiasm, sluggishness, apathy, social withdrawal and disinterest; the anxiety sub-scale assesses tension, worry, and symptoms of anxiety (Zigmond \& Snaith, 1983). Responses for each question vary and are scaled over 3 points from 0 to 3 , with a score of 3 reflecting greater severity. Subscale scores range from 0 to 21 , with a cut-off point score of 11 or more on either subscale considered to be significant for the presence of moderate to severe clinical anxiety or depression symptoms (Montazeri et al., 2000). A total score on the HADS (HADS-T) of 15 was used to define cases of clinical distress. This cut-off point score has been found to have a sensitivity and specificity of greater than $80 \%$ in oncology patients (Mitchell, Meader, \& Symonds, 2010). In this study, the Cronbach's coefficients for the total HADS score was .91, the HADS-A subscale was .85, and the HADS-D subscale was .89. HADS scores were dependent variables; these included the HADS-T, HADS-A and 
HADS-D scores. There are numerous reports in the literature of the use of the HADS with cancer outpatients (Mitchell et al., 2010).

4. Brief COPE. The Brief Coping Inventory contains 28 items and 14 subscales, consisting of two items each, to measure how often respondents use certain strategies to cope with stress. Responses to the items are on a four-point Likert scale. A higher score indicates greater use of a specific coping strategy. Consistent with published research, the subscales were grouped into three coping strategies: problem-focused coping or active (active coping, planning, instrumental support, religion), emotional coping (venting, positive reframing, humor, emotional support, acceptance) and avoidant coping (selfdistraction, denial, behavioral disengagement, self-blame, substance use) (Carver, 1997). The brief COPE scale has good internal consistency; test-retest reliability and concurrent validity are well established. Reliability coefficients for the 14 subscales in cancer patients are reported to range from .50 (venting) to .90 (substance use) (Shapiro et al., 2010). In this study, the Cronbach's coefficients were .85 for the active-coping subscale, .78 for the emotional coping subscale, and .74 for the avoidant coping subscale. The scores on each subscale and the total score were used as independent variables.

5. MSPSS. The 12 item Multidimensional Scale of Perceived Social Support is designed to measure three sources of perceived social support, that from family, friends, and significant others (Zimet, Dahlem, Zimet, \& Farley, 1988). The MSPSS uses a sevenpoint Likert scale ranging from 1, very strongly disagree, to 7, very strongly agree. Higher scores on each of the subscales indicate higher levels of perceived support, and a sum of the three scales yields an overall satisfaction with perceived support score. The MSPSS has good internal reliability coefficients for the subscales; the Cronbach's 
coefficients for the family, friends, and significant other subscales are $0.85,0.86$, and 0.82, respectively (Cicero, Lo Coco, Gullo, \& Lo Verso, 2009). In this study, the Cronbach's coefficients for the family, friends, and significant other subscales were 0.95 , 0.96 , and 0.91 , respectively. There are numerous reports of the use of the MSPSS with cancer patients in the literature. The total score and the scores for each subscale were used as independent variables.

6. STAI. The State Trait Anxiety Inventory measures state and trait aspects of anxiety using two, 20 self-report item scales arranged in Likert scales (Spielberger, Gorsuch, \& Lushene, 1970). Responses for each question are scaled over four points, ranging from 1, not at all, to 4 , very much so. Scores range from 20 to 80 , with a higher score indicating a higher level of state or trait anxiety. Anxiety scores between 20 and 60 were classified as 'low' to 'moderate', and 61-80 as 'high'. The STAI State scale measures how the subject feels at the given moment as characterized by feelings of apprehension, nervousness and worry, whereas the trait scale measures differences in anxiety proneness (Maxwell et al., 2000). Trait anxiety generally does not fluctuate over time, while there typically is an increase in state anxiety levels when one perceives a stressful situation as threatening or dangerous. Each scales' scores were used separately. In this study, the Cronbach's coefficient for the STAI State scale was 0.95 and for the STAI Trait scale was .96 . The score on the STAI State inventory scale was used as a dependent variable reflecting distress; the score on the STAI Trait scale was used as an independent variable. 7. RS-14. The Resilience Scale measures the ability to recover from a stressful situation and conceptualizes resilience as five interrelated attributes: perseverance, selfreliance, meaningfulness, existential aloneness, and equanimity. It uses a 7-point Likert 
rating scale ranging from 1 , strongly disagree, to 7 , strongly agree, with a higher score response indicating higher levels of resilience. Studies published using the RS-14 have reported Cronbach's coefficient over .80. Item-item correlation ranges are reported from $.37-.75$, with the majority in the $.50-.70$ range (Wagnild, 2009). The Cronbach's coefficient in this study was .95 . While there are no specific reports of the use of the RS14 in women with breast cancer, there are several reports validating the use of the RS-14 in patients with other cancers. The total score was used as an independent variable.

8. MLQ. The Meaning in Life Questionnaire assesses two dimensions of meaning in life, presence of meaning and search for meaning. The Presence of Meaning subscale measures the subjective sense that one's life is meaningful, whereas Search for Meaning subscale measures the drive and orientation toward finding meaning in one's life (Steger, Frazier, Oishi, \& Kaler, 2006). The MLQ has 10 items rated on a 7-point Likert scale with responses ranging from 1 , absolutely untrue, to 7, absolutely true. The MLQ has good reliability and test-retest stability. Both subscales have demonstrated good internal consistency with reported coefficients of $.86-.88$ (Steger \& Shin, 2010). In this study, the Cronbach's coefficient for the Presence of Meaning subscale was 0.91 and for the Search for Meaning subscale was .93. Steger and Shin (2010) report that the Presence of Meaning subscale positively correlates with well-being and negatively with anxiety and depression, while the Search for Meaning subscale positively correlates with psychological distress (Steger \& Shin, 2010). The scores on each subscale were used as independent variables. 


\section{Data Analysis}

The investigator screened completed instruments to analyze the extent of missing data. The investigator made a decision on a per case basis as to whether each respondent met criteria; those who did not would have had their responses excluded. In regards to missing data, the investigator evaluated each subject on a per case basis. The subject's responses were still included if the investigator determined data was missing at random. If a subject only answered some of the questions, answered some questions before stopping the survey, or did not return all of the instruments, those responses were not included in data analysis.

\section{Statistical Methods}

The use of several different parametric and nonparametric tests provided a profile of distress in this population. Descriptive statistics were used to analyze demographic characteristics. Chi-square tests were performed to examine for any sample differences among participants at the three sites.

Descriptive statistics were used to determine the prevalence of distress present in women during the breast diagnostic experience. Scores were evaluated on the total score and the two subscales of the HADS (HADS-T, HADS-D, and HADS-A) and the STAI State scale.

Independent t-tests, ANOVA, and Pearson correlations were used to examine the relationships among distress and demographic characteristics, satisfaction with medical care, coping, social support, trait anxiety, resilience, meaning in life. Independent t-tests were calculated using 3 measures of distress, the two subscales of the HADS (HADS-D, and HADSA) and the STAI State scale, and dichotomous demographic characteristics; ANOVA was used with the same distress measures and the nondichotomous demographic characteristics. 
Correlations were calculated using 3 measures of distress, the two subscales of the HADS (HADS-D, and HADS-A) and the STAI State scale, and age, the total score on the RS-14, the total score on the PSQ-18, the total score and scores on the three subscales of the brief COPE (problem-coping, active-coping, and avoidant-coping), the total score and scores on the three subscales of the MSPSS (family support, friend support, and significant other support), STAI Trait scale, and the two subscales of the MLQ (search for meaning and presence of meaning). Independent t- tests were used to examine differences between women aged young and old in regards to distress. Independent t-tests were calculated with age as the grouping variable to examine the score for the two subscales of the HADS (HADS-D and HADS-A) and the STAI State scale.

Independent $t$ - tests were used to examine for differences between women with and without elevated distress and age, satisfaction with medical care, coping method, social support, trait anxiety, resilience, and meaning of life. Those experiencing elevated levels of distress were categorized by score HADS-D and the total score on the STAI State scale. The independent ttests were calculated with level of distress as the grouping variable to examine age, total score on the RS-14, the total score on the PSQ-18, the total score and scores on the three subscales of the brief COPE (problem-coping, active-coping, and avoidant-coping), the total score and scores on the three subscales of the MSPSS (family support, friend support, and significant other support), STAI Trait scale, and the two subscales of the MLQ (search for meaning and presence of meaning). Chi-Square tests were calculated with level of distress as the grouping variable to examine for differences based on the presence of children in the home, level of education, relationship status, employment status, a family history of breast cancer, a personal history of any cancer, and history of a prior abnormal mammogram or breast biopsy. 
Multiple regression was used to evaluate which independent variables were the most efficacious predictors of distress. The use of dependent variables depended on the measurement of the outcome of interest, distress, as measured by indicators of anxiety and depression on the STAI, HADS-A and HADS-D. Based on the analysis of the literature, anxiety was expected to be present; it would have been possible that there would be no measurable signs of depression present. After determining the prevalence of distress present on the measures of distress, two dependent variables were chosen: STAI State Scale and the HADS-D. Each dependent variable was used in a separate model to evaluate factors predictive of those with clinically elevated levels of anxiety or depression. The selection of the predictor variables of interest was based on significant $p$-values from the Pearson correlations, independent t-tests, and Chi-Square tests reflecting their relationship with the dependent variable. Since including more than 6 predictor variables in a regression analysis rarely produces a substantial increase in the model's accuracy and poses the greater the potential for multicollinearity, the number of selected predictor variables entered into the initial models was 6 (Tabachnick \& Fidell, 2007).

\section{Methods to Assure Rigor}

\section{Power Analysis}

Data analysis was conducted using SPSS 17.0 for Windows. The required sample size was estimated based on medium effect size, a desired power of .80, a level of significance of .05, and 6 predictor variables. Using a regression formula based on these criteria $(50+(8)(6))$, it was estimated that a minimum of 98 subjects was needed. Using an independent t-test formula with the same criteria, a minimum of 126 subjects was needed. To assure rigor, the more stringent criteria of 126 subjects was used as a guideline for recruitment of subjects for this study. 


\section{Chapter 4}

\section{Results}

This chapter describes the results of the quantitative data analyses. The first section summarizes the demographic characteristics of the participants. The remainder of the chapter describes the outcome of the quantitative analysis associated with each research question. The prevalence of distress is defined, differences in women who are young and old and have higher levels of distress are delineated, and the predictors of distress in women undergoing breast diagnostic evaluation are evaluated.

\section{Demographic Characteristics}

Descriptive statistics were used to analyze demographic characteristics and Chi-square tests were performed to examine for sample differences among the three sites. Demographic data are presented in Table 1. There were no significant differences in age, the presence of children in the home, level of education, relationship status, or employment status among the three groups. One hundred twenty eight women (128/212) completed the questionnaires, yielding a response rate of $60.4 \%$.

The age of participants ranged from 18 to 89 years with a mean of 55.21 years $(S D=$ 12.21). Roughly one-third (32.8\%) of the women were younger than 50 years old. Ninety-eight (76.6\%) of the women were married or living with a partner, whereas $30(23.4 \%)$ of the women were without a partner, being widowed, divorced, or currently single. The women were nearly divided on level of education. Sixty-nine participants (53.9\%) either did not finish high school or had completed a high school education; 59 women $(46.1 \%)$ had obtained a college or graduate degree. Full-time or part-time employment was held by 64 (49.0\%) of the women, 9 (7.1\%) were unemployed or homemakers, and $41(32.0 \%)$ retired. Fourteen (10.9\%) reported their 
employment status as other while no one reported being on sick leave. Fifty-two women (40.6\%) had a previous abnormal mammogram and nearly three-quarters $(73.1 \%, 38 / 52)$ of these women reported undergoing a previous breast biopsy. Thirty-eight participants $(29.7 \%)$ reported a family history of breast cancer; while 26 women (20.3\%) stated that they had a personal history of a cancer other than breast cancer.

\section{Research Questions}

\section{Question 1}

To determine the prevalence of distress present in women during the breast diagnostic experience scores were analyzed on the STAI State scale and HADS scales. The reported level of distress is shown in Table 2. The average overall score on the STAI State scale was 44.20 (SD 15.186) with scores ranging from 20 to 80 . Anxiety scores between 20 and 60 were classified as "low" to "moderate", and 61-80 as "elevated", indicating the presence of clinical anxiety. In this cohort, 18 women, or $14.3 \%$ had elevated scores indicating the presence of clinical anxiety. The overall average score on the HADS-T was 13.34 with a range of 1 to 31 . HADS-T levels indicating the presence of distress existed for 46 , or $35.9 \%$ of the women. Analysis of the HADS subscales scores revealed that the average overall score for the HADS-A was 8.37 (SD 4.242) with $30.5 \%(n=39)$ of women having clinical anxiety, while the average overall score for the HADS-D was $4.97(S D 4.433)$ with $13.3 \%(\mathrm{n}=17)$ having clinical depression.

Of the 18 women having elevated anxiety scores on the STAI State scale, 8 had elevated scores indicating clinical depression on the HADS-D and 10 did not (Table 3). This difference did not exist in regards to the HADS-A and HADS-D scores. Scores on the HADS-A and STAI were highly correlated $(\mathrm{r}=.752, p<.001)$. Of those 18 women, $15(83.3 \%)$ had clinical anxiety on the HADS-A and only $3(16.7 \%)$ did not. The HADS-A did identify 21 more women than the 
STAI State scale as having clinical anxiety; however, the discrepancy between those with clinical anxiety and those with clinical depression was not as great. Of the 17 women with clinical depression, 13 (76.5\%) were identified on the HADS-A as having clinical anxiety and 4 $(23.6 \%)$ were not.

\section{Question 2}

Independent t-tests, ANOVA, and Pearson correlations were used to determine if there were significant relationships among distress and demographic characteristics, satisfaction with medical care, coping method, social support, and personality factors. Distress was measured using the scores on the STAI State scale and the subscale scores on the HADS (HADS-D and HADS-A).

Using a purposefully devised tool, demographic characteristics collected included age, level of education, employment status, having children under the age of 18 in the home, relationship status, a family history of breast cancer, a personal history of any cancer, and history of a prior abnormal mammogram or breast biopsy. Several significant relationships existed between demographic factors and distress (Table 4). There was a significant relationship between age and the score on the HADS-A, with younger women overall reporting more anxiety $(\mathrm{r}=-.232, p=.008)$. Relationship status was significantly related to score on the STAI State scale $(\mathrm{F}=3.326, p=.044)$ and the HADS-A scale $(\mathrm{F}=3.326, p=.013)$. Women who were single had the highest STAI State mean scores $(M=49.86, S D=7.814)$, with those who were widowed having the lowest mean scores $(M=30.25, S D=14.587)$. On the HADS-A, women who were widowed again had the lowest mean scores $(M=3.63, S D=2.825)$, while all other groups had mean scores between 7.57 and 9.62. Education was significantly related to scores on the HADSD and HADS-A scales. Women with less than a high school education had a mean score on the 
HADS-D of 10.2 while all other groups had mean scores ranging from 4.00 (graduate school degree) to 5.42 (high school graduate). The pattern was nearly identical in regards to the HADSA. Women with less than a high school education had a mean score on the HADS-A of 14.8 while all other groups had mean scores ranging from 7.91 (college graduate) to 8.21 (graduate school). Employment status was significantly related to the scores on the HADS-D ( $\mathrm{F}=4.94, p<$ .001). Women who reported working part-time or were retired had lower mean scores on the HADS-D $(M=2.47, S D=3.482 ; M=3.80, S D=4.106)$, compared to women who were not employed $(M=9.88, S D=5.111)$. Having children under age 18 present in the home was not related to distress.

Women who reported having a history of having an abnormal mammogram, a personal history of other cancer, or a family history of breast cancer were did not more likely to report higher distress than women without this medical history. Women who had undergone a prior breast biopsy had significantly higher score on the HADS-A $(\mathrm{t}=-2.227, p=.028)$ than women who had not previously had a biopsy.

There was a significant relationship between satisfaction with medical care, as reflected on the total score on the PSQ-18, and every measure of distress evaluated (Table 5). Lower levels of satisfaction with medical care correspond with higher levels of anxiety and depression. Sixty-one women $(47.7 \%)$ reported seeking information from a source outside of the health care team regarding an aspect of the experience. Significant differences existed in the level of anxiety as measured by the STAI State scale between women seeking information and those who did not $(\mathrm{t}=1.921, p=.05)$. The mean STAI State score for those who sought information was $46.87(\mathrm{n}=$ 61) while the mean STAI State score for those who did not seek information was $41.76(n=67)$. Of the 61 who reported seeking information from another source, $40(65.6 \%)$ reported using the 
Internet as a source of information. However, there were no significant relationships between the use of the Internet as a source of information and level of distress.

There were no significant relationships between the length of the diagnostic evaluation period and any measure of distress. Five women reported a diagnostic period greater than 120 days; otherwise, the mean diagnostic period was 14.17 days ( $S D$ 16.3, range $0-60)$. Six women reported a diagnostic interval of one day. Of these six women, three (50\%) had clinically elevated anxiety symptoms and four had clinically elevated depression symptoms (66\%). Two of these women had clinically elevated anxiety and depressive symptoms.

The relationship between the measures of distress and coping method was evaluated using scores on the three subscales of the brief COPE, active, emotional, and avoidant coping (see Table 6). Significant relationships existed between the use of avoidant coping strategies and every measure of distress, with those using avoidant coping reporting higher levels of distress. There were no significant relationships between using active coping or emotional coping strategies and any measure of distress.

The relationship between the measures of distress and perceived social support was evaluated using the total score and the scores on the three subscales of the MSPSS, support from family, friends, and significant others (Table 7). Every relationship was significant between the measures of distress and the measures of social support. In this population, distress decreased as levels of perceived social support increased.

The relationship between the measures of distress and personality factors was evaluated using STAI Trait scale, the total score on the RS-14, and the two subscales of the MLQ (search for meaning and presence of meaning (Table 8). Every relationship was significant at $p<.001$ between distress and three of the four assessed personality factors, including trait anxiety, 
resilience, and the presence of meaning. In this population, distress increased as levels of trait anxiety increased. Scores on the STAI Trait scale ranged from 20 to $74(M 39.85, S D=13.463)$, which is comparable to levels in the general population (Maxwell et al., 2000). Ten (7.8\%) of the women had scores on the STAI Trait scale over 60; of these 10, 8 had clinically elevated anxiety. Of the 18 women with clinically elevated anxiety, 8 (44.4\%) had STAI Trait scores over 60. Perceived resilience and how full women felt their lives were decreased distress. Significant correlations existed between search for meaning and all of the measures distress.

\section{Question 3}

Independent $\mathrm{t}$ - tests using the total score and scores for the two subscales of the HADS (HADS-T, HADS-D and HADS-A) and the STAI State scale were used to examine if any significant differences existed between younger and older aged women in level of distress experienced. There were no significant differences in scores between the two age groups in the overall level of distress reported by participants (Table 9).

A Chi-square test was performed to determine if age had any impact on whether a woman had scores on the STAI State scale, HADS-A, or HADS-D that were significant for the presence of moderate to severe clinical anxiety or depression (Table 10). On the HADS-D scale, 16 of the 17 women (94.1\%) scoring an 11 or higher, signaling clinical depression, were over the age of $50\left(\chi^{2}=6.449, p=.011\right)$

\section{Question 4}

Independent $\mathrm{t}$ - tests and Chi-Square tests were used to examine differences between women with and without elevated distress and age, satisfaction with medical care, coping method, social support, trait anxiety, resilience, and meaning of life. As previously described, those experiencing high levels of distress were categorized by assessing the scores on the HADS- 
D subscales and the total score on the STAI State scale. Since $76.5 \%$ of the women identified as having clinically elevated symptoms on the HADS-A and HADS-D were the same women, analysis of the HADS-S results were omitted from this analysis as the populations were analogous. Independent t-tests were calculated with level of distress as the grouping variable to examine age, total score on the RS-14, the total score on the PSQ-18, the scores on the three subscales of the brief COPE (active, emotion and avoidant coping), the total score and scores on the three subscales of the MSPSS (family support, friend support, and significant other support), STAI Trait scale, and the two subscales of the MLQ (search for meaning and presence of meaning).

Chi-Square tests were calculated with level of distress as the grouping variable to examine for differences based on the presence of children in the home, level of education, relationship status, employment status, a family history of breast cancer, a personal history of any cancer, and history of a prior abnormal mammogram or breast biopsy. Three variables, level of education, relationship status, and employment status, were recoded due to cell counts less than 5 in several cases. Level of education was divided into those with a high school education or less and those with a college education. Relationship status was divided into those living with others, either married or in a partnership arrangement, and those living alone. Employment status was divided into those who work in any capacity and those who are not working.

There were significant differences on several measures between women with and without elevated levels of anxiety as measured by the STAI State scale (Table 11 and Table 12). Women with elevated levels of anxiety had mean scores that were significantly lower in satisfaction with medical care, perceived support from friends and family, use of active coping strategies, resilience, and the presence of meaning in their lives. This cohort had mean scores that were 
significantly higher on levels of trait anxiety and the use of avoidant coping strategies and were more likely to report a personal history of cancer.

In comparison, several of these same differences existed between women with and without elevated levels of depression as measured by the HADS-D scale (Table 13 and Table 14). Women with high levels of depression had mean scores that were significantly lower in satisfaction with medical care, perceived support from friends, resilience, and the presence of meaning in their lives. This cohort had mean scores that were significantly higher on the use of avoidant coping strategies and level of trait anxiety. The women scoring higher on depression had an overall mean trait anxiety score of 56.12, which was higher than the population mean of 39.85. There was no relationship between depressive symptoms and perceived family support, the use of active coping strategies, or the personal history of cancer that existed in the cohort with high levels of anxiety.

\section{Question 5}

Multiple regression was used to evaluate which independent variables were the most efficacious predictors of distress in women undergoing a breast diagnostic evaluation for suspected cancer. Based on the previously discussed analysis of the prevalence and level of distress experienced by this population, two dependent variables were chosen: the STAI State Scale and the HADS-D. Each dependent variable was used in a separate model to evaluate factors predictive of those with clinical or elevated levels of anxiety or depression.

The selection of the predictor variables of interest was based on significant $p$-values reflecting their relationship with the dependent variable from the Pearson correlations, independent t-tests, and Chi-Square tests. Therefore, initial independent variables of interest for the predictor model for anxiety were perception of social support from friends, significant others, 
family, and total support; use of avoidant, emotion, and active coping; satisfaction with medical care, information seeking behavior, and personality characteristics, including trait anxiety, resilience and presence of meaning and search for meaning in one's life. After the preliminary analyses for multicollinearity, normality, linearity, and significance were conducted, all the factors except trait anxiety were removed. Table 15 summarizes the multiple regression analysis results. Trait anxiety alone accounted for $71 \%$ of the variance in the STAI State scale score (Multiple $\mathrm{R}=0.842, \mathrm{~F}(1,124)=306.897, p<0.001)$.

To evaluate the influence of variables other than trait anxiety on state anxiety level, a predictor model was performed with the same variables: perception of social support from friends, significant others, family, and total support; use of avoidant, emotion, and active coping; satisfaction with medical care, information seeking behavior, and personality characteristics, including resilience and presence of meaning and search for meaning in one's life. After the preliminary analyses were conducted, three factors were significant: resilience, avoidant coping, and presence of meaning in one's life. This model, however, was not as efficacious, accounting for only $38.7 \%$ of the variance in the STAI State score (Multiple $\mathrm{R}=0.633, \mathrm{~F}(3,123)=27.694, p<0.001)$.

Initial independent variables of interest for the predictor model for depression were employment status, education, perception of social support from friends, family, significant others, and total support, use of avoidant coping, satisfaction with medical care, and personality characteristics, including trait anxiety, resilience, search for meaning in one's life and presence of meaning in one's life. After conducting the preliminary analyses for multicollinearity, normality, linearity, and significance, eight variables, education, social support from family, significant others, and total support, resilience, employment status, search for meaning in one's 
life, and use of avoidant coping, were removed. Table 16 summarizes the multiple regression analysis results. The four remaining variables, trait anxiety, satisfaction with medical care, presence of meaning in one's life, and social support from friends, together accounted for $66 \%$ of the variance in the HADS-D score (Multiple $\mathrm{R}=0.814, \mathrm{~F}(4,123)=60.449, p<0.001)$. Each of these variables was statistically significant, although the associated $R 2$ change was moderate in some cases. As with anxiety, trait anxiety accounted for most of the variance in depression scores $(R 2=.738, p<.001)$.

To evaluate the influence of variables other than trait anxiety on depression, a predictor model was performed with the same variables: employment status, education, perception of social support from friends, family, significant others, and total support, use of avoidant coping, satisfaction with medical care, and personality characteristics, including resilience, search for meaning in one's life and presence of meaning in one's life. After conducting the preliminary analyses, five variables, education, social support from family, significant others, and total support, and employment status were removed. Without trait anxiety, two other personality variables, search for meaning in one's life and resilience, and avoidant coping, emerged as significant; while presence of meaning in one's life and social support from friends remained constant. This model was not as efficacious in predicting the HADS-D score, accounting for $34.2 \%$ of the variance in the HADS-D score (Multiple $\mathrm{R}=0.606, \mathrm{~F}(5,122)=60.06, p<0.001$ ). 


\section{Chapter 5}

The focus of this study was to determine the prevalence of distress and evaluate predictors of distress in women undergoing breast diagnostic evaluation. This chapter presents a discussion of study findings in relation to these aims. This discussion includes an interpretation of results, study limitations, implications for nursing practice, and recommendations for future research.

\section{Discussion}

\section{Prevalence of Distress}

The results of this study validate the presence of distress, manifested as the existence of symptoms of anxiety and depression, throughout the diagnostic period in women undergoing a breast evaluation. This suggests that for those diagnosed with a malignancy, the distress associated with the beginning of the cancer trajectory, which is generally thought of as being the time of receiving a definitive diagnosis, actually begins during the diagnostic evaluation period. Indeed, the average mean score on the STAI State scale of 44.2 was higher than the reported reference average of 34.2 for the general population (Spielberger et al., 1970). It is equivalent or higher than the mean score that has been reported in women in the immediate period after receiving a breast cancer diagnosis and in those undergoing breast cancer treatment with chemotherapy and radiation following surgical intervention (Dolbeault et al., 2009; Fafouti et al., 2010; Schreier \& Williams, 2004). It also validates reports that women experience anxiety prior to their first breast cancer consultation, directly influenced by the thought of having cancer and the impact a diagnosis could have on their lives (Ando et al., 2011; Hegel et al., 2006; Mertz et al., 2012; Okazaki et al., 2009). 
There were differences in the degrees of distress the women experienced. $14.1 \%$ of the women had a level of anxiety that exceeded the cut-off point for a clinically defined anxiety disorder on the STAI State scale with mean scores that were higher than those for patients with a diagnosed anxiety disorder (Kennedy, Schwab, Morris, \& Beldia, 2001; Kvaal, Ulstein, Nordhus, \& Engedal, 2005). When the HADS-A is used as the screening tool, 21 more women were identified as having elevated anxiety levels, increasing the percentage of those with a potential clinical anxiety disorder to $33.3 \%$.

The difference between the STAI State scale and the HADS-A in the number of women identified could be attributed to differences in the content of the scales. Results of a systematic analysis evaluating use of the HADS-A in cancer patients suggests that only three items in the HADS-A assess emotions resulting from a distinct experience while the remainder assess emotions related to trait anxiety (Luckett et al., 2010). Another explanation for these differences is the cut-off score used in this study to identify potential cases for either scale may not appropriate.

There were two unexpected findings in relationship to the prevalence of distress. The first unexpected finding was the number of women (17, or 13.3\%) with symptoms of clinically elevated depression. The existence of this level of depression had not been previously reported in the literature by researchers who have used the HADS-D as a screening tool during the diagnostic period (Andrykowski et al., 2002; Harcourt et al., 1999; Lampic et al., 2001; Lebel et al., 2003; Potter, 2007). It is plausible that the findings here reflect an underlying difference in the actual expression of distress in this population. Other explanations are that the women had clinical depression prior to the diagnostic evaluation period or that other events in their lives were related to depressive symptoms and that completion of the HADS-D was capturing the 
symptomatic response to all life events and not just symptoms related to the diagnostic experience. However, given the prevalence of depression in women is $10.2 \%$, then the percentage reporting depressive symptoms here is slightly higher ("Current Depression Among Adults --- United States, 2006 and 2008," 2013).

The second unexpected finding was that the women who reported clinically elevated anxiety and depression were not necessarily the same women. This is surprising since these two symptoms are often thought to be comorbid (Bidstrup, Johansen, \& Mitchell, 2011; Howell, 2010). Of the 18 women in the cohort with elevated clinical anxiety scores on the STAI State scale, 10 did not have elevated depression scores on the HADS-D. Nine women reported symptoms of elevated depression without an elevation in anxiety symptoms. Again, while this may be an underlying difference in the expression of distress, another explanation is that the women who only had clinical depression had those symptoms prior to the diagnostic evaluation period that were captured by completion of the HADS-D.

Why there is a difference in the identified women with clinically elevated anxiety and depression is not completely clear. Age accounted for some of the difference. While younger age correlated with higher anxiety, 16 of the 17 women with elevated depression were over the age of 50. Older women may feel that they have fewer resources to cope with a cancer diagnosis, perhaps because of decreased availability of family or the presence of co-morbid conditions. The women with clinically elevated symptoms could have had those symptoms prior to the diagnostic evaluation period reflecting preexisting life situations that were partially responsible for the reported symptoms. Alternatively, it could be as simple as different women were predominantly experiencing clinically elevated anxiety or depression, reflecting fundamental differences in their response to the experience. 


\section{Predictors of Distress}

Personality factors, including trait anxiety, resilience, searching for meaning in one's life, and the presence of meaning in one's life, had a substantial influence on the level of distress. In this cohort, distress increased as levels of trait anxiety increased and perceived resilience and how full of meaning women felt their lives were decreased. If one takes into account these aspects of inner strength combined with the impact of education, social support, and satisfaction with medical care, it appears that when faced with the seriousness of the potential diagnosis, level of distress is based upon a woman's personality and her evaluation of whether she feels she has the inner and external resources to enable her to adapt to life with a diagnosis of cancer.

Trait anxiety had the strongest impact on level of distress. Overall, as level of trait anxiety increased, women had higher levels of anxiety and depression. Of the 10 women with high levels of trait anxiety, 8 had clinically elevated anxiety. It is not surprising that women with higher trait anxiety respond to undergoing a biopsy by having heightened distress. These are women who have the tendency to react when confronted with a new or threatening situation with distress, including anxiety and depressive symptoms (de Vries et al., 2009). A potential diagnosis of breast cancer is threatening, and women with higher trait anxiety are experiencing more distress than those with lower trait anxiety. The STAI scales do appear to have been able to discriminate between underlying or personality and situational symptoms. For the 18 women with clinically elevated anxiety, only $8(44.4 \%)$ had STAI Trait scores over 60 , indicating that a majority of women who experienced situational anxiety did not have underlying anxiety tendencies.

Resilience correlated with level of distress, with women with higher resilience experiencing less anxiety and fewer depressive symptoms. This relates back to the premise that 
level of distress is based upon a woman's evaluation of whether she feels she has the inner resources to cope with a diagnosis of cancer. Resilient women have less distress because they feel they have the personal strengths and capabilities to cope effectively or to fight if diagnosed with cancer. Indeed, there are reports that when there is a lack of an attitude of being a so-called "fighter" in in women with breast cancer, there is greater distress (Montazeri et al., 2000).

There were significant correlations between search for meaning and all of the measures of overall distress. Women who are still trying to find their place in life and feel that their life is lacking or unsettled had increased distress levels. Since meaning in life is regarded as an indicator of personal well-being, this relationship is not unexpected as a lower sense of purpose has been related to increased distress in adverse experiences (Steger et al., 2006; Steger \& Shin, 2010). Here, women may view a potential cancer diagnosis as another deterrent to their finding their place in life or see their situation as hopeless, and have increased anxiety and depression.

As anticipated, there was a substantial correlative relationship between age and anxiety. Overall, younger women reported more anxiety, which is not surprising. There had previously been some documentation of this relationship in the literature in both women undergoing a diagnostic evaluation as well as those with breast cancer and ovarian cancer (Arden-Close, 2008; Drageset \& Lindstrom, 2005; Mertz et al., 2012). The results here affirming an association between younger age and increased anxiety supports the health care team's need and responsibility to provide younger women with adequate support. The fact, though, that there was no relationship between the presence of children in the home and distress was surprising given that the younger women were more likely to have children in the home and the numerous reports of the concern women with children had regarding the impact that a cancer diagnosis would have on the children's future (Chappy, 2004; Demir et al., 2008; Logan et al., 2006). 
Older age was a significant influence on a woman's level of depression. Women over age 50 comprised $94 \%$ of those who had clinically elevated depression. As discussed earlier, older women may feel that they will not have the resources to cope with a cancer diagnosis, perhaps because of decreased availability of family or the presence of co-morbid conditions. It is plausible that the women with clinically elevated symptoms had those symptoms prior to the diagnostic evaluation period reflecting preexisting life situations that were partially responsible for the reported symptoms.

A woman's medical history did not have as great of an impact on distress levels as had been expected based on reports in the literature. Women who had undergone a prior breast biopsy were more likely to be anxious. It is possible that women with a history of a breast lesion that was potentially cancerous or a benign breast disease that places them at an increased risk for breast cancer estimate the risk of the current lesion being cancer as higher, increasing their distress level. Then, the question needs to be asked why a family history of breast cancer was not related to distress as the perceived the risk of having cancer should have been higher. It is plausible that this difference could be directly attributed to the previous experience of undergoing a biopsy, especially if the previous experience was a negative one, given the role of satisfaction with medical care on anxiety and depressive symptoms.

Satisfaction with medical care was highly influential on the experience of undergoing a breast evaluation. Women who were more satisfied with their health care had decreased reports of anxiety and depression. As discussed earlier, women gave accounts in the qualitative literature of some very unsupportive behaviors, where women felt that the health care team did not treat them with respect and should have recognized their experience as unique. Since some items on the PSQ-18 ask respondents to gauge whether members of the health care team treat 
them in ways such as courteously, personally, and attentively, it is not surprising that a relationship between satisfaction with care and distress was confirmed.

Perceived adequacy of information may have had some influence on satisfaction with health care. Approximately half of the women spent time seeking information from a source outside of the health care time regarding an aspect of the diagnostic experience; those with clinically elevated anxiety were more likely to participate in seeking outside information. It is unclear the direction of this relationship. The possibility exists that using the Internet as an information source may alleviate some distress by helping women feel that they have the level of information that they need to be prepared. Conversely, reading information that is not pertinent to a woman's particular situation may heighten anxiety levels.

Education was significantly related to distress. Women with less than a high school education were likely to have higher anxiety and depressive symptoms than those with at least a high school education. This may relate back to satisfaction with health care and information related behaviors. As discussed earlier, women with a lower level of education may have experienced more distress due to a lack of access to information, not fully understanding the information they were given and having fewer resources (Andrykowski et al., 2002; Northouse et al., 1995; Novy et al., 2001; Olsson et al., 1999). In women with breast cancer, higher distress levels in those with lower educational levels was explained by the fact that women with higher education levels were more likely to seek and obtain the information they wanted related to cancer and its related aspects (Vahdaninia, Omidvari, \& Montazeri, 2010).

Employment status was significantly related to the presence of depressive symptoms, with women working full-time experiencing more depressive symptoms. It may be possible that the women were concerned about the impact that a diagnosis would have on their role in the 
workplace, and worried about the logistics associated with taking a leave of absence from work and being able to reassign work responsibilities.

Women engaged in a wide range of coping behaviors during the diagnostic period. The use of specific coping strategies influenced distress levels, with the use of avoidance, aimed at distancing oneself from the stressor, associated with higher anxiety and depressive symptoms. On every measure, the use of avoidant coping strategies, such as denying the experience is occurring or blaming one's self, or participating in alternative activities, such as smoking, sleeping, using drugs or drinking more alcohol, correlated significantly with higher levels of anxiety and depression. These findings coincide with those previously discussed of women undergoing a breast biopsy and support reports that women with breast and other cancers using avoidance are more distressed (Howell, 2010; Montazeri et al., 2000).

Why is the use of avoidant coping deleterious? By denying the experience is occurring, those who use avoidant coping behaviors may be hindering their problem-solving abilities. This relates back to the premise that undergoing preparative psychological processes, rehearsing what life with breast cancer would be like and considering practical issues, such as the logistics associated with taking a leave of absence from work and being able to reassign work responsibilities or who would potentially be able to provide childcare, are useful and necessary. Contrary to some previous descriptions in the literature, there was an inverse relationship in this study between social support and distress (Allen et al., 2008; Drageset \& Lindstrom, 2003; Liao et al., 2008). In this population, there was strong evidence that distress levels increased as levels of perceived social support decreased, with every relationship being significant between the measures of distress and the measures of social support. It could be inferred that those with better social support dealt better with experience by having solid 
networks that could support them during this difficult situation. A strength of this study was using a tool that assessed perceived social support from three separate sources of support, a significant other, family and friends, as opposed to evaluating the social network as a whole. Therefore, by allowing women to delineate how support is given differently, for example, between a son and a best friend, it allowed for a more accurate gauge of the relationship between their distress and perceived social support.

Consequently, the strongest relationship was not between the women and their significant other or family, but between the women and their friends. This relationship especially persisted when evaluating those with the elevated anxiety and depressive symptoms and perceived support. In those cohorts, only support from friends was significant across every measure of distress with increased perceived friend support being associated with a decrease in distress, particularly in those with elevated depressive symptoms. It is possible that women view social support from family as expected or an obligation, while support from friends has a greater impact as it is an unconditional, voluntary relationship, and therefore holds more importance (Arora, Finney-Rutten, Gustafson, Moser, \& Hawkins, 2007; Cicero et al., 2009). Another explanation may be that women in this cohort had already relied on friend support, seeking out those in their network who had previously undergone a biopsy and that these "similar others" were already providing a valuable source of informational and emotional support (Lebel et al., 2003; Thorne et al., 1999). The perception of family support may be dampened by a woman's worries regarding the impact a cancer diagnosis would have on her family. A woman may also feel that her family members and significant other may not be able to offer them support at the level they desire because of the quality of their relationship. 
For those living alone, the distress level was vastly different dependent on the woman's circumstance. Being single correlated with higher levels of anxiety; while widows experienced significantly lower levels of anxiety symptoms. This dichotomy has not been reported before and the reason is not completely clear. It could relate back to the role of friendships and social support. The mean age of the widows was 66.23 years. One could hypothesize that the widows may have a more established social network or friends provided a source of support during the diagnostic period in the absence of a significant other. Another possible explanation might be that the loss of the husband and experiencing bereavement has given the widow better coping abilities.

There were considerable differences in duration of the diagnostic period, ranging from a single day upwards to 6 months. Overall, there were no significant relationships between the length of the diagnostic evaluation period and any measure of distress, implying that a consistent level of distress persisted throughout the diagnostic period. There appears to be the possibility that a speedier diagnosis may be associated with heightened distress. Of the six women reporting a diagnostic interval of one day, three had clinically elevated anxiety and four had clinically elevated depression symptoms. A possible explanation for the symptoms in this cohort is that undergoing those preparative psychological processes described earlier is useful and necessary. Unfortunately, the sample size for this group was not large enough to draw any meaningful statistical conclusions.

In this study, a small cohort of women had levels of anxiety that were above the cut-off point for clinically defined anxiety disorder. These women were likely to be less satisfied with their medical care, have a lower level of perceived support from friends, use avoidant coping strategies, and have specific personality traits, including being less resilient, lacking a presence 
of meaning in their lives and, most importantly, possessing a high level of trait anxiety. The only significant difference between these women and those without elevated levels of anxiety was having a personal history of cancer. This suggests that for cancer survivors, facing the prospect of experiencing another cancer trajectory is particularly distressful (Schnur et al., 2008). A woman's age, though, was not a factor in determining if a woman experienced elevated levels of anxiety.

The profile of the women with clinically elevated depression was essentially the same. Women with elevated levels of depression also had specific personality traits, including possessing a high level of trait anxiety, being less resilient, and lacking a presence of meaning in their lives, being less satisfied with their medical care, using avoidant coping strategies, and having a lower level of perceived support from friends. The women who were depressed, however, were more likely to have a high school education or less. As discussed earlier, women with a lower level of education may experience distress due to a lack of access to information or feeling that they do not have the resources to cope in the event of a cancer diagnosis.

Given the role that personality traits appears to play in managing the distress associated with a breast diagnostic evaluation, it was not unexpected that the most efficacious predictor of distress, for both anxiety and depressive symptoms, is trait anxiety. Trait anxiety was the only factor in the model for predicting anxiety symptoms on the STAI State scale and accounted for most of the variance in the model for predicting depressive symptoms on the HADS-D. Three other factors played a lesser role in predicting depressive symptoms: satisfaction with medical care, presence of meaning in one's life, and social support from friends. As discussed earlier, each of these had an impact on whether a woman had clinically elevated symptoms. 


\section{Application of Findings to UIT}

The new insight into the distress experienced by women undergoing a breast diagnostic evaluation provided by this study lends credence to the applicability of UIT as a framework for studying women experiencing a breast biopsy. Within the context of UIT, a woman appraised the potential outcome associated with a breast diagnostic evaluation as a threatening, resulting in distress. Distress, manifested as anxiety and depressive symptoms, was present throughout the diagnostic period.

In applying UIT to the prediction of distress, several key tenets of UIT did influence the existence of distress. According to UIT, the level of distress would depend on how a woman appraised and managed the event (Mishel, 1990). Appraisal is based not only the interpretation of the potential severity of the illness and impact on a woman's life, but also on her past experiences and personality. The distress experienced by women in this study was related to a previous history of having a breast biopsy, the presence of meaning in their lives, and personality factors, including trait anxiety and resilience. Managing the event prompts coping efforts directed at reducing the level of distress. In UIT, coping through active confrontation is the best means to attain the lowest level of distress possible. In this study, women who used avoided coping methods to manage uncertainty associated with the situation experienced higher levels of distress.

Two other key antecedents influence distress in UIT, social support and credible authority. Social support functions to buffer the effects of the event through receiving information and advice (Mishel, 1988). Those women with better perceived social support did experience decreased distress levels. Women rely on the credible authority provided by the health care team as the primary source of information regarding the evaluation process. Here, 
women with a higher level of satisfaction with their medical care did experience decreased distress, affirming that health care providers can directly decrease distress by providing information and a supportive environment (Mishel et al., 2002).

\section{Recommendations}

Because of the existence of distress associated with the diagnostic evaluation period, there is a need to assess for distress and provide appropriate interventions for women undergoing a breast diagnostic evaluation. The National Comprehensive Cancer Network (NCCN) has developed guidelines recommending that psychological distress should be recognized and treated at all stages of disease, beginning at the initial visit (Holland et al., 2010). The results of this study support that the factors that predict distress during the diagnostic evaluation period are the same as those for women in the immediate period after diagnosis. Therefore, the initial visit should be defined as the time when a woman presents for a diagnostic test that will offer a confirmed diagnosis, such as a breast biopsy, and not at the time of receiving a definitive diagnosis or the first consultation after receiving a definitive diagnosis.

The initial step is to apply the evidence gained from this study to the development of clinical guidelines for distress screening during the diagnostic evaluation period. Tools recommended for screening use need to be able to identify the presence of anxiety and depression, being mindful that certain factors, including personality traits, age, satisfaction with medical care, and perceptions of support, influence the level of distress, and quantify the level of distress, appropriately identify those women who are in need of support that is more intensive. Since trait anxiety is the factor most strongly associated with distress, using a screening tool that captures this insight into a woman's personality is essential. For use in clinical practice, tools that take only a few minutes to complete are preferred. 
Assessment for distress alone will not improve a woman's distress or overall quality of life; assessment must be followed by determining the woman's needs and implementing interventions aimed at mediating distress levels. The following recommended set of nursedelivered interventions are similar to those suggested for newly diagnosed breast cancer patients and are comprised of information combined with psychosocial support focusing on how to cope with distress related to undergoing biopsy.

The first recommendation is to provide women with information that is relevant to that point in time. This includes the purpose of diagnostic tests, the type of information tests provide, what to expect during a test, any pre- and post-procedural care, and when test results will be available. Information should be at the desired level of depth and at the proper level of understanding. Every center providing diagnostic services should assure that women have access to a contact person with whom to speak who can provide and clarify information. Conversations should be supplemented by giving women written materials and directing those that are interested to specific Internet sites for further information. Videos may be used prior to a biopsy describing what to expect during the procedure. Finally, women should be given information on how to make or change appointments, directions to the facility, parking facilities, and the availability of transportation if needed.

Women should be offered psychosocial support in a caring environment. There is a need to discuss what the woman may be experiencing emotionally and acknowledge that distress is expected when undergoing a diagnostic evaluation. Since empathic listening plays an important role in the relief of distress, nurses can be extremely helpful by listening to concerns, allowing women to express their feelings, and encouraging the use of social support networks. Women should be guided in identifying helpful support persons and the level of information they want to 
share with others about their situation. Nurses can encourage women to consider having a supportive person present during visits. For those waiting between procedures, at least one routine follow-up telephone call may be useful to check on the woman, screen again for distress, and discuss any concerns.

Nurses can enhance a woman's daily quality of life during the evaluation period by assisting women to identify their coping behaviors and provide anticipatory guidance regarding the use of appropriate coping mechanisms. Women who relate using avoidant-coping strategies should be encouraged to use problem-focused or emotion-focused strategies, which directly assist women in managing the distress they are experiencing. Therefore, women should be encouraged to participate in activities such as talking with a friend, prayer, meditating, listening to music or exercising. It should be emphasized to avoid activities such as smoking more, sleeping more, increasing antianxiety medication use, or drinking more alcohol more often. Nurses can explore with women strategies they feel they have used successfully in past stressful situations and encourage them to use those strategies that assisted them with reducing stress previously if these are problem or emotion- focused.

A woman's satisfaction with medical care may be improved by facilitating communication among healthcare providers. For those needing assistance with navigating the health care system, nurses can help a woman select health care agencies and providers for performing diagnostic procedures, being mindful of potential limits by an insurance company or other third party payer, and assist in completing financial applications. For referrals and subsequent testing, nurses can aid in making subsequent appointments and transferring records and copies of tests to the next provider. These interventions are particularly important in the 
community hospital setting, where women are more likely to receive fragmented services (Allen et al., 2008; Robinson-White et al., 2010).

There is a need to utilize specific interventions dependent upon the degree of distress present. If a woman is identified as having clinically elevated symptoms or her screening profile places her at a heightened risk for experiencing clinically elevated symptoms, she needs a more thorough assessment and evaluation for appropriate treatment. This may include a follow-up telephone call to see if how she is doing, an additional appointment, or a referral to pastoral care, social work or mental health services for more formal individual counseling. Short-term medication use with hypnotics, antidepressants, and anxiolytics may be considered in some situations to assist women in managing symptoms.

Women need to be appropriately triaged after diagnosis. For those diagnosed with cancer, providers should follow the NCCN recommendations. When a diagnosis of cancer is given, it can be overwhelming, increasing distress and affecting the ability to think critically and make decisions (Harcourt et al., 1999; Iwamitsu et al., 2005). Some women may benefit from an extra appointment or counseling session focusing on providing psychosocial support and discussing the woman's concerns and additional support can be given in an effort to assist with making informed decisions about treatment options. If the woman experienced elevated distress during the diagnostic period, this should be recognized so that appropriate screening and further intervention can be initiated.

Protocols need to be developed and put in place for women who receive a benign diagnosis since the distress experienced during the diagnostic period may persist in some women, affecting future mammography behaviors and quality of life. Nurses can discuss the type of benign breast disease, describe if it poses a risk for subsequently developing breast 
cancer, and outline an appropriate follow-up plan with the recommended intervals for subsequent clinical breast exams and mammography. Women should be provided with a means to follow-up either at the center performing the evaluation or with a primary care provider if they have further questions or need someone with whom to talk and for any care needs. Those who are still distressed may need further intervention, such as counseling so they may talk about their concerns stemming from the experience.

The results of this study provide evidence supporting funding for a case management or navigation program aimed at providing women support during the diagnostic evaluation period. In routine clinical practice, time constraints often lead to little or no time for a physician to offer detailed information or assist a woman with managing her distress. Since registered nurses possess the knowledge, skills and clinical judgment necessary for delivering all of the interventions described here, a key component of a program is having a nurse available to women throughout the diagnostic phase and to fulfill the pivotal roles described here of providing information, facilitating communication with health care providers, and offering emotional support.

Implementing a navigation program for all women undergoing a diagnostic evaluation may not be easy to accomplish. While comprehensive cancer centers are required to offer the services of a patient navigator, at least $50 \%$ of biopsies are performed outside of comprehensive cancer care centers (Gutierrez et al., 2008; Williams et al., 2011). In a non-navigated setting, it would not be an uncommon scenario for a woman to be notified by letter that a screening mammogram was abnormal, for her to make her own arrangements for a diagnostic mammogram, receive the results from a primary care physician, and if a referral is needed for a surgical biopsy, she must select a surgeon and is responsible for making that appointment. 
Whose responsibility, then, does providing a navigation program become when women receive fragmented services? Does the responsibility lie with the facility performing the diagnostic mammogram or biopsy, the surgeon performing the biopsy or the primary care physician?

Budgetary restrictions could be a potential issue in providing navigation services to women during the diagnostic period. In hospitals that see a lower volume of women, for example, a facility that performs 100 biopsies per year, it may be that the only cost-efficient model utilizes a part-time nurse navigator or has a full-time nurse navigator who has other job responsibilities beyond providing pre-diagnosis services.

Determining the relative benefits of pre-diagnosis navigation in relation to cost of providing care can be difficult to determine. Two measures that are used to directly evaluate program outcomes are time to diagnosis and treatment and the adherence rate for completing recommended diagnostic tests. An indirect measure of a navigation program's benefit may be better utilization of physician services. Anxious women tend to make more telephone calls to clinics or hospitals, using more physician time (Pedersen, Sawatzky, \& Hack, 2010). In routine clinical practice, time constraints often lead to little or no time for a physician to offer detailed information or assist a woman with managing her distress. A nurse navigator providing information and support would have women better prepared for their appointments and making decisions, allowing physicians to make more efficient use of their time with the woman (Gilbert et al., 2011).

With Medicare's new funding program, facility reimbursement is partially based on patient satisfaction. Navigated women should not only experience less distress, but through promoting a better experience, the services should translate into higher satisfaction ratings through better-coordinated care and improved satisfaction with the quality of health care 
received (Korber, Padula, Gray, \& Powell, 2011; Markossian \& Calhoun, 2011). A nurse navigator making a routine follow-up telephone call to women to see how they are doing should directly translate to improved patient satisfaction; patients appreciate someone checking up on how they are doing (Rush, 2012).

\section{Limitations}

A few limitations affect the conclusions drawn from study findings. The first is that the volume of patients at one of the sites was much smaller than the volume at the other two sites; the result was that two sites accounted for most of the study participants. For three variables of interest, education, employment status, and relationship status, the original variables were recoded into fewer groups so that cell sizes were adequate enough to perform the appropriate statistical analyses. The impact on distress of a rapid diagnosis could not be evaluated as the cohort of women who received a one day diagnosis was too small to allow for an adequate comparison. Another study limitation was the use of a cross-sectional design with self-reported instruments, so uncontrolled or unexplored variables may have influenced study results. Since a history of psychiatric illness was not an exclusion criterion, it is possible that the number of women with a history of comorbid psychiatric problem was high. Finally, results are limited to conclusions based on the responses of women who chose to participate. The forty percent of the women who did not return the study packets may have differed in their level of distress and had fundamental differences in their personality or other variable of interest that may have influenced distress levels and study findings. 


\section{Directions for Further Research}

The results of this study delineate a number of areas for further research. One of the first areas requiring further research is to determine which tools are the best to use for screening women for distress during the diagnostic evaluation period. While there are several methods recommended for screening in cancer patients, there are not specific tools recommended for use in women undergoing a diagnostic evaluation. The tools used need to be able to accurately gauge the distress experienced, yet short enough to be clinically useful.

While the STAI scales used here performed acceptably, each subscale is 20 items long and requires a 10 minute time commitment. The HADS-T, with its' two subscales, is shorter, at 14 items, and can be completed in 5 minutes. These tools could be used to measure the performance of simple analogue scales, including the Distress Thermometer, a tool endorsed by the NCCN for use in cancer patients, in gauging distress level and identifying women at risk for elevated symptoms (Hegel et al., 2006). For any tools selected, the best cut-off scores for identifying potential women at risk need to be defined.

To provide evidence as to the most effective means to mediate distress, intervention studies using a longitudinal design need to be performed to test the outcomes of nursing interventions to mediate distress. These nursing interventions should encompass providing psychosocial support, facilitating communication with healthcare providers, providing counseling regarding coping behaviors, and educating women regarding all aspects of the diagnostic evaluation period. Studies should also be done to test the outcomes of targeted interventions for those women experiencing elevated anxiety and depressive symptoms.

Further research is needed into how to best implement or expand navigation program services for women undergoing a diagnostic evaluation, particularly in the community hospital 
setting, and to evaluate the overall impact of a navigation program on distress levels. There is a need to determine how to address logistical issues of moving women through the diagnostic process when services are received at different locations, how to best administer services when women are receiving fragmented care, and how to examine the cost effectiveness of program delivery, especially in facilities that see lower volumes of women.

Given the number of women who spent time seeking information regarding the diagnostic experience from a source outside of the health care system, further exploration is needed into women's information-seeking behaviors. These studies should more fully explore women's sources of information, their preferences for sources of information, how they use the additional information, and how this information influences distress.

With the impact that perceptions of social support has on distress, further research should explore how to best have women utilize social support within the context of undergoing a diagnostic evaluation. It would be helpful to know how women use their social resources and if increasing perceptions of support diminishes distress. Since it appears that the perception of friend support has the greatest impact on distress, knowing more about how women view friendships as a distress-lowering resource and the efficacy of interventions to assist women who have low perceptions of friend support would fill a knowledge gap. If it is recommended that women bring a husband or significant other with them to consultations, it would be useful to study the role of bringing a friend with them when results are being discussed and establish if this is effective in the reduction of distress.

The hint of the higher levels of depression and anxiety in those who had a diagnostic interval of one day suggests that there may be a relationship between this practice and distress. Since the cohort of women in this study was not large enough to make any definitive 
conclusions, further exploration is needed into the impact of a rapid diagnosis on distress, especially for those diagnosed with cancer. If it is found that their experience differs, then protocols need to be designed and tested to support women who are diagnosed in this manner, such as a seeing the woman more than once before asking her to make a treatment decision.

\section{Conclusion}

The results of this study provide a greater understanding of the experience of women undergoing a breast evaluation. The presence of distress, manifested as symptoms of anxiety and depression, was validated throughout the diagnostic period. A clear profile emerged of factors that influence distress and of the woman who is more likely to have clinically elevated anxiety and depressive symptoms. Level of distress appears to be based upon a woman's personality and her evaluation of whether she feels she has the inner and external resources to able to adapt to life with a diagnosis of cancer.

This better understanding of factors that influence distress levels provides the evidence for basing interventions aimed at managing associated distress. Because of the existence of distress, there is a need to assess for distress and provide appropriate interventions to women undergoing a breast diagnostic evaluation. A key component of a program is having a nurse available to women throughout the diagnostic phase to fulfill the pivotal roles providing information, facilitating communication with health care providers, and offering emotional support. The outcome is having the woman ready to face the future regardless of the outcome of the biopsy. 


\section{References}

Allen, J. D., Shelton, R. C., Harden, E., \& Goldman, R. E. (2008). Follow-up of abnormal screening mammograms among low-income ethnically diverse women: findings from a qualitative study. Patient Education And Counseling, 72(2), 283-292. doi: 10.1016/j.pec.2008.03.024

Anderson, J., Ganz, P. , Bower, J.E. , \& Stanton, A.L. (2012). Quality of Life, Fertility Concerns, and Behavioral Health Outcomes in Younger Breast Cancer Survivors: A Systematic Review Journal of the National Cancer Institute. doi: doi: 10.1093/jnci/djr541

Ando, N., Iwamitsu, Y., Kuranami, M., Okazaki, S., Wada, M., Yamamoto, K., . . Miyaoka, H. (2009). Psychological characteristics and subjective symptoms as determinants of psychological distress in patients prior to breast cancer diagnosis. Supportive Care In Cancer, 17(11), 1361-1370. doi: 10.1007/s00520-009-0593-7

Ando, N., Iwamitsu, Y., Kuranami, M., Okazaki, S.., Nakatani, Y., Yamamoto, K., ... Miyaoka, H. (2011). Predictors of psychological distress after diagnosis in breast cancer patients and patients with benign breast problems. Psychosomatics, 52(1), 56-64. doi: 10.1016/j.psym.2010.11.012

Andrykowski, M. A., Carpenter, J. S., Studts, J. L., Cordova, M. J., Cunningham, L. L. C., Beacham, A., . . McGrath, P. (2002). Psychological impact of benign breast biopsy: a longitudinal, comparative study. Health Psychology, 21(5), 485-494.

Arden-Close, E., Gidron Y., \& Moss-Morris, R. . (2008). Psychological distress and its correlates in ovarian cancer: a systematic review. Psycho-Oncology, 17, 1061-1072. doi: 10.1002/pon.1363 
Aro, A. R., Pilvikki Absetz, S., van Elderen, T. M., van der Ploeg, E., \& van der Kamp, L. J. (2000). False-positive findings in mammography screening induces short-term distress breast cancer-specific concern prevails longer. European Journal Of Cancer, 36(9), 1089-1097.

Arora, N. K., Finney-Rutten, L. J., Gustafson, D. H., Moser, R., \& Hawkins, R. P. (2007). Perceived helpfulness and impact of social support provided by family, friends, and health care providers to women newly diagnosed with breast cancer. Psycho-Oncology, 16(5), 474-486. doi: 10.1002/pon. 1084

Atkinson, P.A., Martin, C.R. \& Rankin, R.A. (2009). Resilience revisited. Journal of Psychiatric and Mental Health Nursing, 16, 137-145. doi: 10.1111/j.1365-2850.2008.01341.x

Baldacchino, D., \& Draper, P. (2001). Spiritual coping strategies: a review of the nursing research literature. Journal of Advanced Nursing, 34(6), 833-841.

Barton, M. B., Morley, D. S., Moore, S., Allen, J. D., Kleinman, K. P., Emmons, K. M., \& Fletcher, S. W. (2004). Decreasing women's anxieties after abnormal mammograms: A controlled trial. Journal of the National Cancer Institute, 96(7), 529-538. doi: 10.1092/jnci/djh083

Bidstrup, P. E., Johansen, C., \& Mitchell, A. J. (2011). Screening for cancer-related distress: Summary of evidence from tools to programmes. Acta Oncologica, 50(2), 194-204. doi: $10.3109 / 0284186 X .2010 .533192$

Birney, M. (1995). Stress responses in women experiencing a breast biopsy for cancer. Dissertation Abstracts International: Wayne State University. 
Bradley, P. K., Berry, A., Lang, C., \& Myers, R. E. (2006). Getting ready: developing an educational intervention to prepare African American women for breast biopsy. Association of Black Nursing Faculty Journal, 17(1), 15-19.

Brewer, N.T., Salz, T., \& Lillie, S.E. (2007). Systematic review: the long-term effects of falsepositive mammograms. Annals of Internal Medicine, 146(7), 502-510.

Brocken, P., Prins, J. B. , Dekhuijzen, R., \& van der Heijden, H. (2012). The faster the better?-A systematic review on distress in the diagnostic phase of suspected cancer, and the influence of rapid diagnostic pathways. Psycho-Oncology, 21(1), 1-10. doi: 10.1002/pon.1929

Brodersen, J. , Thorsen, H., \& Kreiner, S. (2007). Validation of a condition-specific measure for women having an abnormal screening mammography. Value Health, 10(4), 294-304.

Bury, M. (1982). Chronic illness as biographical disruption. Sociology in Health and Illness, $4(2), 167-182$.

Cancer Facts and Figures: 2012. (2012, February 10, 2013). from http://www.cancer.org/Research/CancerFactsFigures/CancerFactsFigures/cancer-factsfigures-2011

Carver, C. S. (1997). You want to measure coping but your protocol's too long: Consider the Brief COPE. International Journal of Behavioral Medicine, 4(1), 92-100.

Cesario, S. K., Nelson, L. S., Broxson, A., \& Cesario, A. L. (2010). Sword of Damocles Cutting Through the Life Stages of Women With Ovarian Cancer. Oncology Nursing Forum, 37(5), 609-617. doi: 10.1188/10.onf.609-617

Chappy, S. L. (2004). Women's experience with breast biopsy. American Association of Operating Room Nurses Journal, 80(5), 885-901. 
Chen, C. C., David, A., Thompson, K., Smith, C., Lea, S., \& Fahy, T. (1996). Coping strategies and psychiatric morbidity in women attending breast assessment clinics. Journal of Psychosomatic Research, 40(3), 265-270.

Ching, S. S. Y., Martinson, I. M., \& Wong, T. K. S. (2009). Reframing: psychological adjustment of Chinese women at the beginning of the breast cancer experience. Qualitative Health Research, 19(3), 339-351. doi: 10.1177/1049732309331867

Cicero, V., Lo Coco, G., Gullo, S., \& Lo Verso, G. (2009). The role of attachment dimensions and perceived social support in predicting adjustment to cancer. Psycho-Oncology, 18(10), 1045-1052. doi: 10.1002/pon.1390

Cimprich, B., So, H., Ronis, D. L., \& Trask, C. (2005). Pre-treatment factors related to cognitive functioning in women newly diagnosed with breast cancer. Psycho-Oncology, 14(1), 7078. doi: 10.1002/pon.821

Clutton, S., Buckley, B., \& Pakenham, K. I. (1999). Predictors of emotional well-being following a false positive breast cancer screening result. Psychology \& Health, 14(2), 263.

Connor, K.M. , \& Davidson, J. (2003). Development of a new resilience scale: The ConnorDavidson Resilience Scale (CD-RISC). Depression \& Anxiety (1091-4269), 18(2), 76.

Current Depression Among Adults --- United States, 2006 and 2008. (2013). Retrieved 03/09/2013, from http://www.cdc.gov/mmwr/preview/mmwrhtml/mm5938a2.htm?s_cid=mm5938a2_e\%0 $\mathrm{d} \% 0 \mathrm{a}$

De Grasse, C. E., Hugo, K., \& Plotnikoff, R. C. (1997). Supporting women during breast diagnostics. Canadian Nurse, 93(9), 24-30. 
de Vries, J., van der Steeg, A. F., \& Roukema, J. A. (2009). Trait anxiety determines depressive symptoms and fatigue in women with an abnormality in the breast. British Journal of Health Psychology, 14(1), 143-157. doi: 10.1348/135910708X310200

Deane, K. A., \& Degner, L. F. (1998). Information needs, uncertainty, and anxiety in women who had a breast biopsy with benign outcome. Cancer Nursing, 21(2), 117-126.

Degner, L. F., Hack, T., O'Neil, J., \& Kristjanson, L. J. (2003). A new approach to eliciting meaning in the context of breast cancer. Cancer Nursing, 26(3), 169-178.

DeKeyser, F. G., Wainstock, J. M., Rose, L., Converse, P. J., \& Dooley, W. (1998). Distress, symptom distress, and immune function in women with suspected breast cancer. Oncology Nursing Forum, 25(8), 1415-1422.

Demir, F., Donmez, Y. C., Ozsaker, E., \& Diramali, A. (2008). Patients lived experiences of excisional breast biopsy: a phenomenological study. Journal of Clinical Nursing, 17(6), 744-751. doi: 10.1111/j.1365-2702.2007.02116.x

Devins, G. M., Bezjak, A., Mah, K., Loblaw, D. A. , \& Gotowiec, A. P. (2006). Context moderates illness-induced lifestyle disruptions across life domains: a test of the illness intrusiveness theoretical framework in six common cancers. Psycho-Oncology, 15(3), 221-233. doi: 10.1002/pon.940

Dey, P., Bundred, N., Gibbs, A., Hopwood, P., Baildam, A., Boggis, C., . . W Woodman, C. (2002). Costs and benefits of a one stop clinic compared with a dedicated breast clinic: randomised controlled trial. BMJ: British Medical Journal, 324(7336), 507.

Dingley, C.E., Bush, H. A., \& Roux, G. (2000). Inner strength: A Concept Analysis. Journal of Theory Construction \& Testing, 4(2), 30-35. 
Dolbeault, S., S., Cayrou, A., Brédart, L., Viala A., B., Desclaux, P., Saltel, .. P., Dickes. (2009). The effectiveness of a psycho-educational group after early-stage breast cancer treatment: results of a randomized French study. Psycho-Oncology, 18(6), 647-656. doi: 10.1002/pon. 1440

Drageset, S., \& Lindstrom, T. C. (2003). The mental health of women with suspected breast cancer: the relationship between social support, anxiety, coping and defence in maintaining mental health. Journal of Psychiatric \& Mental Health Nursing, 10(4), 401409. doi: 10.1046/j.1365-2850.2003.00618.x

Drageset, S., \& Lindstrom, T. C. (2005). Coping with a possible breast cancer diagnosis: demographic factors and social support. Journal of Advanced Nursing, 51(3), 217-226. doi: $10.1111 / \mathrm{j} .1365-2648.2005 .03495 . x$

Drageset, S., Lindstrom, T. C., \& Underlid, K. (2010). Coping with breast cancer: between diagnosis and surgery. Journal of Advanced Nursing, 66(1), 149-158. doi: $10.1111 /$ j.1365-2648.2009.05210.x

Ell, K., Vourlekis, B., Lee, P., \& Xie, B. (2007). Patient navigation and case management following an abnormal mammogram: a randomized clinical trial. Preventive Medicine, 44(1), 26-33.

Fafouti, M., Paparrigopoulos, T., Zervas, Y., Rabavilas, A., Malamos, N., Liappas, I., \& Tzavara, C. (2010). Depression, anxiety and general psychopathology in breast cancer patients: a cross-sectional control study. In Vivo 24(5), 803-810.

Ferrante, J., Chen, P., \& Kim, S. (2008). The effect of patient navigation on time to diagnosis, anxiety, and satisfaction in urban minority women with abnormal mammograms: a 
randomized controlled trial. Journal Of Urban Health: Bulletin Of The New York Academy Of Medicine, 85(1), 114-124.

Fridfinnsdottir, E. B. (1997). Icelandic women's identifications of stressors and social support during the diagnostic phase of breast cancer. Journal of Advanced Nursing, 25(3), 526531.

Gil, K. M., Mishel, M. H., Belyea, M., Germino, B., Porter, L. S., \& Clayton, M. (2006). Benefits of the uncertainty management intervention for African American and White older breast cancer survivors: 20-month outcomes. International Journal of Behavorial Medicine, 13(4), 286-294. doi: 10.1207/s15327558ijbm1304_3

Gil, K. M., Mishel, M. H., Belyea, M., Germino, B., Porter, L. S., LaNey, I. C., \& Stewart, J. (2004). Triggers of uncertainty about recurrence and long-term treatment side effects in older African American and Caucasian breast cancer survivors. Oncology Nursing Forum, 31(3), 633-639. doi: 10.1188/04.onf.633-639

Gilbert, J. E., Green, E., Lankshear, S., Hughes, E., Burkoski, V., \& Sawka, C. (2011). Nurses as patient navigators in cancer diagnosis: review, consultation and model design. European Journal of Cancer Care, 20(2), 228-236. doi: 10.1111/j.1365-2354.2010.01231.x

Giske, T., \& Artinian, B. (2008). Patterns of 'balancing between hope and despair' in the diagnostic phase: a grounded theory study of patients on a gastroenterology ward. Journal of Advanced Nursing, 62(1), 22-31. doi: 10.1111/j.1365-2648.2007.04523.x

Guadalupe, K. (2010). Understanding a meningioma diagnosis using Mishel's theory of uncertainty in illness. British Journal of Neuroscience Nursing, 6(2), 77-82. 
Gutierrez, J. C., Hurley, J. D., Housri, N., Perez, E. A., Byrne, M. M., \& Koniaris, L. G. (2008). Are many community hospitals undertreating breast cancer? Annals Of Surgery, 248(2), 154-162. doi: 10.1097/SLA.0b013e31816c4030

Haas, J., Kaplan, C., McMillan, A., \& Esserman, L. J. (2001). Does timely assessment affect the anxiety associated with an abnormal mammogram result? Journal of Women's Health \& Gender-Based Medicine, 10(6), 599-605. doi: 10.1089/15246090152543184

Harcourt, D., Rumsey, N., \& Ambler, N. (1999). Same-day diagnosis of symptomatic breast problems: psychological impact and coping strategies. Psychology, Health \& Medicine, 4(1), 57-71. doi: 10.1080/135485099106405

Harding, M., \& McCrone, S. (2011). Psychological distress associated with the diagnostic phase for suspected breast cancer: Systematic review. Journal of Advanced Nursing, 66(11), 2372-2390. doi: 10.1111/j.1365-2648.2010.05439.x

Heckman, B. D., Fisher, E. B., Monsees, B., Merbaum, M., Ristvedt, S., \& Bishop, C. (2004). Coping and anxiety in women recalled for additional diagnostic procedures following an abnormal screening mammogram. Health Psychology, 23(1), 42-48. doi: 10.1037/02786133.23.1.42

Hegel, M. T., Moore, C. P., Collins, E. D., Kearing, S., Gillock, K. L., Riggs, R. L., ... Ahles, T. A. (2006). Distress, psychiatric syndromes, and impairment of function in women with newly diagnosed breast cancer. Cancer, 107(12), 2924-2931.

Hislop, T. Gregory, Harris, Susan R., Jackson, Jeremy, Thorne, Sally E., Rousseau, Eunice J., Coldman, Andrew J., . . Olivotto, Ivo A. (2002). Satisfaction and anxiety for women during investigation of an abnormal screening mammogram. Breast Cancer Research And Treatment, 76(3), 245-254. 
Holland, J. C., Andersen, B. L., Breitbart, W., Compas, B., Dudley, M., Fleishman, S., .. . Zevon, M. A. (2010). Distress management. Journal Of The National Comprehensive Cancer Network, 8(4), 448-485.

Howell, D. (2010). Psychological distress as a nurse-sensitive outcome. In D. M. Doran (Ed.), Nursing Outcomes: State of the Science (2nd ed., pp. 285-358). Sudbury, MA: Jones \& Bartlett.

Iwamitsu, Y., Shimoda, K., Abe, H., Tani, T., Okawa, M., \& Buck, R. (2005). Anxiety, emotional suppression, and psychological distress before and after breast cancer diagnosis. Psychosomatics, 46(1), 19-24.

Kennedy, B. L. , Schwab, J. J., Morris, R. L. , \& Beldia, G. (2001). Assessment of State and Trait Anxiety in Subjects with Anxiety and Depressive Disorders. Psychiatric Quarterly, 72(3), 263.

Keyzer-Dekker, C.., De Vries, J., van Esch, L., Ernst, M. F., Nieuwenhuijzen, G. A. , Roukema, J. A., \& van der Steeg, A. F. . (2012). Anxiety after an abnormal screening mammogram is a serious problem. Breast, 21(1), 83-88.

Korber, S. F., Padula, C., Gray, J., \& Powell, M. (2011). A Breast Navigator Program: Barriers, Enhancers, and Nursing Interventions. Oncology Nursing Forum, 38(1), 44-50. doi: 10.1188/11.onf.44-50

Kvaal, K., Ulstein, I., Nordhus, I. H., \& Engedal, K. (2005). The Spielberger State-Trait Anxiety Inventory (STAI): the state scale in detecting mental disorders in geriatric patients. International Journal of Geriatric Psychiatry, 20(7), 629-634. doi: 10.1002/gps.1330 
Lampic, C., Thurfjell, E., Bergh, J., \& Sjoden, P. O. (2001). Short- and long-term anxiety and depression in women recalled after breast cancer screening. European Journal of Cancer, 37(4), 463-469.

Lauver, D., \& Tak, Y. (1995). Optimism and coping with a breast cancer symptom. Nursing Research, 44(4), 202-207.

Lazarus, R. S. (2006). Emotions and interpersonal relationships: toward a person-centered conceptualization of emotions and coping. Journal Of Personality, 74(1), 9-46. doi: $10.1111 /$ j.1467-6494.2005.00368.x

Lebel, S., Jakubovits, G., Rosberger, Z., Loiselle, C., Seguin, C., Cornaz, C., . . Lisbona, A. (2003). Waiting for a breast biopsy: Psychosocial consequences and coping strategies. Journal of Psychosomatic Research, 55(5), 437. doi: 10.1016/S0022-3999(03)00512-9

Liao, M., Chen, M., \& Chen, S. (2008). Uncertainty and anxiety during the diagnostic period for women with suspected breast cancer. Cancer Nursing, 31(4), 274-283. doi: 10.1097/01.NCC.0000305744.64452

Liao, M., Chen, M., Chen, S., \& Chen, P. (2007). Healthcare and support needs of women with suspected breast cancer. Journal of Advanced Nursing, 60(3), 289-298. doi: 10.1111/j.1365-2648.2007.04410.x

Liao, M., Chen, P., Chen, M., \& Chen, S. (2010). Effect of supportive care on the anxiety of women with suspected breast cancer. Journal of Advanced Nursing, 66(1), 49-59. doi: $10.1111 /$ j.1365-2648.2009.05139.x

Logan, J, Hackbusch-Pinto, R, \& De Grasse, C. E. (2006). Women undergoing breast diagnostics: The lived experience of spirituality. Oncology Nursing Forum, 33(1), 121126. doi: $10.1188 / 06$. ONF.121-126 
Lowe, J. B., Balanda, K. P., Del Mar, C., \& Hawes, E. (1999). Psychologic distress in women with abnormal findings in mass mammography screening. Cancer, 85(5), 1114-1118.

Luckett, T., Butow, P.s N., King, M. T., Oguchi, M., Heading, G., Hackl, N. A., . . Price, M. A. (2010). A review and recommendations for optimal outcome measures of anxiety, depression and general distress in studies evaluating psychosocial interventions for English-speaking adults with heterogeneous cancer diagnoses. Supportive Care In Cancer, 18(10), 1241-1262. doi: 10.1007/s00520-010-0932-8

Lundman, B., Aléx, L., Jonsén, E., Norberg, A., Nygren, B., Santamäki-Fischer, R., \& Strandberg, G. (2009). Inner strength--A theoretical analysis of salutogenic concepts. International Journal of Nursing Studies, In Press, Corrected Proof. doi: 10.1016/j.ijnurstu.2009.05.020

MacFarlane, M. E., \& Sony, S. D. (1992). Women, breast lump discovery, and associated stress. Health Care for Women International, 13(1), 23-32.

Markossian, T. W., \& Calhoun, E. A. (2011). Are breast cancer navigation programs costeffective? Evidence from the Chicago Cancer Navigation Project. Health Policy, 99(1), 52-59. doi: 10.1016/j.healthpol.2010.07.008

Marshall, G.N., \& Hayes, R.D. (1994). Patient Satisfaction Questionnaire Short Form (pp. 1-39). Santa Monica, CA: RAND.

Maxwell, J. R., Bugbee, M. E., Wellisch, D., Shalmon, A., Sayre, J., \& Bassett, L. W. (2000). Imaging-guided core needle biopsy of the breast: Study of psychological outcomes. Breast Journal, 6(1), 53-61. doi: 10.1046/j.1524-4741.2000.98079.x

Mertz, B. G., Bistrup, P. E. , Johansen, C., Dalton, S. O. , Deltour, I., Kehlet, H., \& Kroman, N. (2012). Psychological distress among women with newly diagnosed breast cancer. 
European Journal Of Oncology Nursinghe Official Journal Of European Oncology Nursing Society, 16(4), 439-443. doi: 10.1016/j.ejon.2011.10.001

Meystre-Agustoni, G., Paccaud, F., Jeannin, A., \& Dubois-Arber, F. (2001). Anxiety in a cohort of Swiss women participating in a mammographic screening programme. Journal of Medical Screening, 8(4), 213-219.

Mishel, M. H. (1988). Uncertainty in illness. Image: The Journal of Nursing Scholarship, 20(4), $225-232$.

Mishel, M. H. (1990). Reconceptualization of the uncertainty in illness theory. Image: The Journal of Nursing Scholarship, 22(4), 256-262.

Mishel, M. H. (1997). Uncertainty in acute illness. Annual Review of Nursing Research, 15, 5780.

Mishel, M. H., Belyea, M., Germino, B. B., Stewart, J. L., Bailey, D. E., Robertson, C., \& Mohler, J. (2002). Helping patients with localized prostate carcinoma manage uncertainty and treatment side effects: nurse-delivered psychoeducational intervention over the telephone. Cancer, 94(6), 1854-1866.

Mitchell, A. J., Meader, N., \& Symonds, P. (2010). Diagnostic validity of the Hospital Anxiety and Depression Scale (HADS) in cancer and palliative settings: A meta-analysis. Journal of Affective Disorders, 126(3), 335-348. doi: 10.1016/j.jad.2010.01.067

Montazeri, A., Harirchi, II, Vahdani, M., Khaleghi, F., Jarvandi, S., Ebrahimi, M., \& HajiMahmoodi, M. (2000). Anxiety and depression in Iranian breast cancer patients before and after diagnosis. European Journal of Cancer Care, 9(3), 151-157. 
Montgomery, G. , \& Bovbjerg, D. H. (2004). Presurgery distress and specific response expectancies predict postsurgery outcomes in surgery patients confronting breast cancer. Health Psychology, 23(4), 381-387.

Montgomery, G. H. , David, D., Goldfarb, A.B., Silverstien, J.H., Weltz, C.R., Birk, J.S., \& Bovberg, D.H. . (2003). Sources of anticipatory distress among breast surgery patients. Journal of Medical Screening, 26(2), 153-164.

NAPBC Accreditation. (2012, January 19, 2013). from http://napbcbreast.org/accreditation/accreditation.html

Northouse, L. L., Jeffs, M., Cracchiolo-Caraway, A., Lampman, L., \& Dorris, G. (1995). Emotional distress reported by women and husbands prior to a breast biopsy Nursing Research, 44(4), 196-201.

Northouse, L. L., Tocco, K. M., \& West, P. (1997). Coping with a breast biopsy: How healthcare professionals can help women and their husbands. Oncology Nursing Forum, 24(3), 473480.

Novy, D. M., Price, M., Huynh, P. T., \& Schuetz, A. (2001). Percutaneous core biopsy of the breast: correlates of anxiety. Academic Radiology, 8(6), 467-472.

O'Mahony, M. (2001). Women's lived experience of breast biopsy: a phenomenological study. Journal of Clinical Nursing, 10(4), 512-520.

Okazaki, S., Iwamitsu, Y., Masaru, K., Todoroki, K., Suzuki, S., Yamamoto, K., . . Miyaoka, H. (2009). The psychological responses of outpatient breast cancer patients before and during first medical consultation. Palliative \& Supportive Care, 7(3), 307-314. doi: $10.1017 / \mathrm{S} 147895150999023 \mathrm{X}$ 
Olsson, P., Armelius, K., Nordahl, G., Lenner, P., \& Westman, G. (1999). Women with false positive screening mammograms: how do they cope? Journal of Medical Screening, 6(2), 89-93.

Ong, G., \& Austoker, J. (1997). Recalling women for further investigation of breast screening: women's experiences at the clinic and afterwards. Journal Of Public Health Medicine, 19(1), 29-36.

Pedersen, A. E., Sawatzky, J., \& Hack, T. F. (2010). The Sequelae of Anxiety in Breast Cancer: A Human Response to Illness Model. Oncology Nursing Forum, 37(4), 469-475.

Penrod, J. (2007). Living with uncertainty: concept advancement. Journal of Advanced Nursing, 57(6), 658-667. doi: 10.1111/j.1365-2648.2006.04008.x

Pineault, P. (2007). Breast cancer screening: women's experiences of waiting for further testing. Oncology Nursing Forum, 34(4), 847-853. doi: 10.1188/07.ONF.847-853

Poole, K., Hood, K., Davis, B. D., Monypenny, I. J., Sweetland, H., Webster, D. J., . . Mansel, R. E. (1999). Psychological distress associated with waiting for results of diagnostic investigations for breast disease. Breast, 8(6), 334-338.

Poole, K., \& Lyne, Patricia A. (2000). The 'cues' to diagnosis: describing the monitoring activities of women undergoing diagnostic investigations for breast cancer. Journal of Advanced Nursing, 31(4), 752-758. doi: 10.1046/j.1365-2648.2000.01345.x

Potter, P. J. (2007). Breast biopsy and distress: feasibility of testing a Reiki intervention. Journal of Holistic Nursing, 25(4), 238-248.

Rasmussen, D. M., \& Elverdam, B. (2007). Cancer survivors' experience of time: time disruption and time appropriation. Journal of Advanced Nursing, 57(6), 614-622. 
Rehnberg, G., Absetz, P., \& Aro, A. R. (2001). Women's satisfaction with information at breast biopsy in breast cancer screening. Patient Education \& Counseling, 42(1), 1-8.

Riese, S. (2001). Women's experiences with breast biopsy. Dissertation Abstracts International, 62(3), 194 (AAT 3008789).

Robinson-White, S., Conroy, B., Slavish, K. H., \& Rosenzweig, M. (2010). Patient navigation in breast cancer: a systematic review. Cancer Nursing, 33(2), 127-140.

Roux, G., Lewis, K., Younger, J., \& Dingley, C. (2003). Development and testing of the inner strength questionnaire. Journal of Cultural Diversity, 10(1), 4-5.

Rush, S. K. (2012). Discharge Calls: How One Call Can Make the Difference for Patients, Families, and Reimbursement. Nurse Leader, 10(2), 45-52. doi: 10.1016/j.mnl.2011.07.014

Schnur, J. B., Montgomery, G. H., Hallquist, M. N., Goldfarb, A. B., Silverstein, J. H., Weltz, C. R., , . Bovbjerg, D. H. (2008). Anticipatory Psychological Distress in Women Scheduled for Diagnostic and Curative Breast Cancer Surgery. International Journal of Behavioral Medicine, 15(1), 21-28. doi: 10.1080/10705500701783843

Schreier, A. M., \& Williams, S. A. (2004). Anxiety and quality of life of women who receive radiation or chemotherapy for breast cancer. Oncology Nursing Forum, 31(1), 127-130. doi: 10.1188/04.ONF.127-130

Seckel, M. M., \& Birney, M. H. (1996). Social support, stress, and age in women undergoing breast biopsies. Clinical Nurse Specialist, 10(3), 137-143.

Shapiro, J.P., McCue, K., Heyman, E. N., Dey, T. , \& Haller, H. S. (2010). Coping-Related Variables Associated with Individual Differences in Adjustment to Cancer. Journal of Psychosocial Oncology, 28(1), 1-22. doi: 10.1080/07347330903438883 
Shaw, C. R., Wilson, S. A., \& O'Brien, M. E. (1994). Information needs prior to breast biopsy. Clinical Nursing Research, 3(2), 119-131.

Spielberger, C.D., Gorsuch, R. L., \& Lushene, R. E. (1970). Manual for the State-Trait Anxiety Inventory (Self-evaluation questionnaire). Palo Alto, CA: Consulting Psychologists Press.

Stanton, A. L., \& Snider, P. R. (1993). Coping with a breast cancer diagnosis: a prospective study. Health Psychology, 12(1), 16-23.

Steger, M. F., Frazier, P., Oishi, S., \& Kaler, M. . (2006). The Meaning in Life Questionnaire: Assessing the presence of and search for meaning in life. Journal of Counseling Psychology, 53, 80-93. doi: 10.1037/0022-0167.53.1.80

Steger, M. F., \& Shin, J. Y. . (2010). The relevance of the Meaning in Life Questionnaire to therapeutic practice: A look at the initial evidence. International Forum on Logotherapy, $33,95-104$.

Tabachnick, B.G., \& Fidell, L.S. . (2007). Using Multivariate Statistics (5th ed.). Boston, MA: Pearson.

Thorne, S. E., Harris, S. R., Hislop, T. G., \& Vestrup, J. A. (1999). The experience of waiting for diagnosis after an abnormal mammogram. Breast Journal, 5(1), 42.

Tugade, Michele M., \& Fredrickson, Barbara L. (2007). Regulation of Positive Emotions: Emotion Regulation Strategies that Promote Resilience. Journal of Happiness Studies, $8(3), 311-333$.

Vahdaninia, M., Omidvari, S., \& Montazeri, A. (2010). What do predict anxiety and depression in breast cancer patients? A follow-up study. Social Psychiatry \& Psychiatric Epidemiology, 45(3), 355-361. doi: 10.1007/s00127-009-0068-7 
Wagnild, G. (2009). A review of the resilience scale. Journal of Nursing Measurement, 17(2), 105-113.

Walter, J. M. (2005). Psychoneuroimmunological manifestations of stress in women undergoing breast biopsy. Dissertation Abstracts International, 66(11), 164 (AAT 3196515).

Warner, E. (2011). Clinical practice. Breast-cancer screening. The New England Journal Of Medicine, 365(11), 1025-1032.

Williams, R. T., Yao, K., Stewart, A. K., Winchester, D. J., Turk, M., Gorchow, A., .. . Winchester, D. P. (2011). Needle versus excisional biopsy for noninvasive and invasive breast cancer: report from the National Cancer Data Base, 2003-2008. Annals Of Surgical Oncology, 18(13), 3802-3810. doi: 10.1245/s10434-011-1808-y

Winchester, D. P. (2008). The National Accreditation Program for Breast Centers: A Multidisciplinary Approach to Improve the Quality of Care for Patients with Diseases of the Breast, Breast Journal, pp. 409-411.

Witek-Janusek, L., Gabram, S., \& Mathews, H. L. (2007). Psychologic stress, reduced NK cell activity, and cytokine dysregulation in women experiencing diagnostic breast biopsy. Psychoneuroendocrinology, 32(1), 22-35. doi: 10.1016/j.psyneuen.2006.09.011

Zigmond, A. S., \& Snaith, R. P. (1983). The hospital anxiety and depression scale. Acta Psychiatrica Scandinavica, 67(6), 361-370.

Zimet, G. D., Dahlem, N. W., Zimet, S. G., \& Farley, G. K. (1988). The Multidimensional Scale of Perceived Social Support. Journal of Personality Assessment, 52(1), 30. 
Table 1

Demographic Characteristics

\begin{tabular}{ccccc}
\hline Characteristic & Total & Site 1 & Site 2 & Site 3 \\
$(n=128)$ & $(n=50)$ & $(n=5)$ & $(n=73)$
\end{tabular}

Age

Under 50

Over 50

Relationship status

Single

Married

Separated/ divorced

Living with partner

Widowed

Children in home

Yes

No

Education

Less than high school

High school

College graduate

Graduate degree

Employment

Full-time

Part-time

Not employed

Looking for work

Retired
$42(32.8 \%) \quad 19(38.0 \%) \quad 1(20.0 \%) \quad 22(30.1 \%) \quad \chi 2=1.220$

$86(67.2 \%) \quad 31(62.0 \%) \quad 4(80.0 \%) \quad 51(69.9 \%)$ $(n=73)$ 


$\begin{array}{lllll}\text { Other } & 14(10.9 \%) & 7(14.0 \%) & 0 & 7(9.6 \%)\end{array}$

Prior abnormal mammogram

$\begin{array}{llllll}\text { Yes } & 52(40.6 \%) & 19(38.0 \%) & 1(20.0 \%) & 32(43.8 \%) & \chi 2=1.337 \\ \text { No } & 76(59.4 \%) & 31(62.0 \%) & 4(80.0 \%) & 41(56.2 \%)\end{array}$

Previous breast biopsy

Yes

$$
\begin{array}{llllll}
\text { Yes } & 42(32.8 \%) & 17(34.0 \%) & 1(20.0 \%) & 24(32.9 \%) & \chi 2=.404 \\
\text { No } & 86(67.2 \%) & 33(66.0 \%) & 4(80.0 \%) & 49(67.1 \%) &
\end{array}
$$

Family history breast cancer

$\begin{array}{lccccc}\text { Yes } & 38(29.7 \%) & 12(24.0 \%) & 1(20.0 \%) & 25(34.2 \%) & \chi 2=2.382 \\ \text { No } & 84(65.6 \%) & 36(72.0 \%) & 4(80.0 \%) & 44(60.3 \%) & \\ \text { Unknown } & 6(4.7 \%) & 2(4.0 \%) & 0 & 4(5.5 \%)\end{array}$

Personal history of other cancer

$\begin{array}{llllll}\text { Yes } & 27(21.1 \%) & 15(30.0 \%) & 2(40.0 \%) & 10(13.7 \%) & \chi 2=7.543 \\ \text { No } & 101(78.9 \%) & 35(70.0 \%) & 3(60.0 \%) & 63(86.3 \%) & \end{array}$

$* p<.05 . * * p<.01$ 
Table 2

Level of Distress

\begin{tabular}{lc}
\hline \multicolumn{1}{c}{ Measure } & $n(\%)$ \\
\hline STAI State & $110(85.9)$ \\
$20-60$, Low to moderate & $18(14.1)$ \\
$61-80$, Elevated & \\
HADS-T & $82(64.1)$ \\
$0-15$, Not significant & $46(35.9)$ \\
$16-42$, Elevated & $89(69.5)$ \\
HADS- A & $39(30.5)$ \\
$0-10$, Not significant & \\
$11-21$, Elevated & $111(86.7)$ \\
HADS- D & $17(13.3)$ \\
$0-10$, Not significant & \\
\hline
\end{tabular}


Table 3

Classification on Measures of Distress

STAI State Level

Measure $\quad$ Low to moderate $(n=110) \quad$ Elevated $(n=18)$

HADS-T

Low to moderate

80

2

Elevated

30

16

HADS- A

Low to moderate

86

3

Elevated

24

15

HADS- D

Low to moderate

101

10

Elevated

9

8 
Table 4

Relationships between Distress Measures and Demographic Characteristics

Measure

Characteristic

STAI HADS-A

HADS-D

\begin{tabular}{|c|c|c|c|}
\hline Age & $r=-.155$ & $\mathrm{r}=-.232 * *$ & $\mathrm{r}=-.008$ \\
\hline Age Group & $t=.703$ & $\mathrm{t}=1.863$ & $\mathrm{t}=-1.673$ \\
\hline $\begin{array}{l}\text { Relationship } \\
\text { status }\end{array}$ & $\mathrm{F}=2.526^{*}$ & $\mathrm{~F}=3.326^{*}$ & $F=1.800$ \\
\hline Children in home & $\mathrm{t}=1.445$ & $\mathrm{t}=1.750$ & $t=.674$ \\
\hline Education & $\mathrm{F}=.471$ & $\mathrm{~F}=4.339 * *$ & $\mathrm{~F}=3.632 *$ \\
\hline Employment & $\mathrm{F}=.971$ & $\mathrm{~F}=2.919 * *$ & $\mathrm{~F}=4.94 * *$ \\
\hline $\begin{array}{l}\text { Prior abnormal } \\
\text { mammogram }\end{array}$ & $\mathrm{t}=.211$ & $\mathrm{t}=-1.583$ & $\mathrm{t}=-.908$ \\
\hline $\begin{array}{l}\text { Previous breast } \\
\text { biopsy }\end{array}$ & $\mathrm{t}=.058$ & $\mathrm{t}=-2.227^{*}$ & $\mathrm{t}=-1.221$ \\
\hline $\begin{array}{l}\text { Family history } \\
\text { breast cancer }\end{array}$ & $\mathrm{t}=.440$ & $\mathrm{t}=-.005$ & $t=.616$ \\
\hline $\begin{array}{l}\text { Personal history } \\
\text { of other cancer }\end{array}$ & $\mathrm{t}=1.765$ & $\mathrm{t}=1.129$ & $\mathrm{t}=1.339$ \\
\hline
\end{tabular}

$* p<.05 . * * p<.01$ 
Table 5

Relationships between Distress Measures and Health Care

\begin{tabular}{|c|c|c|c|c|}
\hline Measure & $\begin{array}{c}\text { Satisfaction with } \\
\text { Care }\end{array}$ & $\begin{array}{c}\text { Information } \\
\text { Seeking }\end{array}$ & Internet Use & $\begin{array}{c}\text { Length of Diagnostic } \\
\text { Period }\end{array}$ \\
\hline STAI State & $\mathrm{r}=-.353 * *$ & $\mathrm{t}=1.921^{*}$ & $t=-.762$ & $r=-.100$ \\
\hline HADS-A & $\mathrm{r}=-.249 * *$ & $\mathrm{t}=1.280$ & $\mathrm{t}=-.737$ & $\mathrm{r}=-.090$ \\
\hline HADS-D & $\mathrm{r}=-.458 * *$ & $\mathrm{t}=-1.652$ & $\mathrm{t}=-1.325$ & $r=-.156$ \\
\hline
\end{tabular}

${ }^{*} p<.05 . * * p<.01$ 
Table 6

Correlations between Distress Measures and Coping Methods

\begin{tabular}{|c|c|c|c|}
\hline Measure & Emotional Coping & Avoidant Coping & Active Coping \\
\hline STAI State & .075 & $.373 * *$ & .093 \\
\hline HADS-A & .131 & $.511 * *$ & .082 \\
\hline HADS-D & -.091 & $.258 * *$ & -.071 \\
\hline
\end{tabular}


Table 7

Correlations between Distress Measures and Social Support

\begin{tabular}{|c|c|c|c|c|}
\hline Measure & Family & Friend & Significant Other & Total Support \\
\hline STAI State & $-.291 * *$ & $-.318 * *$ & $-.263 * *$ & $-.335 * *$ \\
\hline HADS -A & $-.260 * *$ & $-.296 * *$ & $-.228 * *$ & $-.300 * *$ \\
\hline HADS- D & $-.369 * *$ & $-.536 * *$ & $-.346 * *$ & $-.478 * *$ \\
\hline
\end{tabular}

${ }^{*} p<.05 .{ }^{* *} p<.01$ 
Table 8

Correlations among Distress Measures and Personality Factors

\begin{tabular}{|c|c|c|c|c|}
\hline Measure & Trait Anxiety & Resilience & $\begin{array}{c}\text { Presence of } \\
\text { Meaning }\end{array}$ & $\begin{array}{l}\text { Search for } \\
\text { Meaning }\end{array}$ \\
\hline STAI State & $.842 * *$ & $-.554 * *$ & $-.501 * *$ & $.332 * *$ \\
\hline HADS-A & $.750 * *$ & $-.520 * *$ & $-.476^{* *}$ & $.387 * *$ \\
\hline HADS-D & $.738 * *$ & $-.647 * *$ & $-.684 * *$ & $.178 *$ \\
\hline
\end{tabular}


Table 9

Differences in Distress between Young and Old Women

\section{Age}

$\begin{array}{lll}\text { Measure } & \text { Under } 50(\mathrm{n}=42) & \text { Over } 50(\mathrm{n}=86)\end{array}$

\begin{tabular}{|c|c|c|c|}
\hline \multirow[t]{2}{*}{ STAI State } & $M=45.55$ & $M=43.53$ & $t(126)=.703$ \\
\hline & $(S D=15.736)$ & $(S D=14.959)$ & \\
\hline \multirow[t]{2}{*}{ HADS-T } & $M=13.52$ & $M=13.24$ & $\mathrm{t}(126)=.189$ \\
\hline & $(S D=7.062)$ & $(S D=8.222)$ & \\
\hline \multirow[t]{2}{*}{ HADS-A } & $M=9.36$ & $M=7.88$ & $t(126)=1.863$ \\
\hline & $(S D=4.400)$ & $(S D=4.103)$ & \\
\hline \multirow[t]{2}{*}{ HADS- D } & $M=4.17$ & $M=5.36$ & $t(126)=-1.673$ \\
\hline & $(S D=3.084)$ & $(S D=4.930)$ & \\
\hline
\end{tabular}


Table 10

Differences in Elevated Distress between Young and Old Women

\begin{tabular}{lccc}
\hline & \multicolumn{3}{c}{ Age } \\
\cline { 2 - 3 } Distress Measure & Under $50(\mathrm{n}=42)$ & Over $50(\mathrm{n}=86)$ & \\
\hline STAI & $8(44.4 \%)$ & $10(55.6 \%)$ & .285 \\
HADS-T & $14(30.4 \%)$ & $32(69.6 \%)$ & .106 \\
HADS-A & $12(30.8)$ & $27(69.2 \%)$ & $6.449 * *$ \\
HADS- D & $1(5.9 \%)$ & $16(94.1 \%)$ & \\
\hline$* p<.05 . * * p<.01$ & & &
\end{tabular}


Table 11

Differences by Anxiety Level on STAI State Scale

\begin{tabular}{|c|c|c|c|}
\hline Characteristic & $\begin{array}{l}\text { Low to moderate }(n=110) \\
\qquad M(S D)\end{array}$ & $\begin{array}{c}\text { Elevated }(n=18) \\
M(S D)\end{array}$ & $\mathrm{t}$ \\
\hline Age & $55.64(12.599)$ & $52.51(9.382)$ & .974 \\
\hline Satisfaction with care & $67.63(.099)$ & $62.22(10.074)$ & $2.302 *$ \\
\hline STAI trait & $36.74(11.292)$ & $58.89(9.486)$ & $7.873 * *$ \\
\hline Resilience & $81.01(13.556)$ & $61.50(18.125)$ & $4.371 * *$ \\
\hline Total support & $73.05(13.153)$ & $62.50(21.109)$ & 2.064 \\
\hline Family & $24.75(4.774)$ & $21.11(6.995)$ & $2.793 * *$ \\
\hline Friend & $23.67(4.882)$ & $19.78(7.175)$ & $2.221 *$ \\
\hline Significant other & $24.62(6.148)$ & $21.61(8.417)$ & 1.454 \\
\hline Total coping & $56.64(13.778)$ & $55.29(11.655)$ & .250 \\
\hline Active coping & $19.80(5.624)$ & $18.88(3.551)$ & $2.185^{*}$ \\
\hline Emotion coping & $22.24(6.902)$ & $19.00(6.021)$ & .912 \\
\hline Avoidant coping & $14.59(4.358)$ & $17.41(4.199)$ & $-2.311^{*}$ \\
\hline Presence of meaning & $28.04(5.543)$ & $17.76(11.377)$ & $2.512 *$ \\
\hline Search for meaning & $17.09(8.383)$ & $15.88(10.700)$ & -.621 \\
\hline
\end{tabular}


Table 12

Demographic Differences by Anxiety Level on the STAI State Scale

\begin{tabular}{lccc}
\hline & Low to moderate $(n=110)$ & Elevated $(n=18)$ & $\chi 2$ \\
Characteristic & $n$ & $n$ &
\end{tabular}

\section{Relationship status}

Alone

With another

Children in home

Yes

No

Education

High school or less

College

Employment

Working

Not Working

Prior abnormal mammogram

Yes

No

Previous breast biopsy

Yes

35

75

No

Family history breast cancer

Yes

32

72

No

Unknown
60

50

28

82

28

82

68

42

42

68

8

10

8

11

16

6

1.774

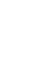




\begin{tabular}{lccc}
\hline Personal history of other cancer & & & \\
Yes & 19 & 8 & $6.862 * *$ \\
No & 91 & 10 & \\
\hline$* p<.05 . * * p<.01$ & & &
\end{tabular}


Table 13

Differences by Depression Level on the HADS-D Scale

\begin{tabular}{|c|c|c|c|}
\hline Characteristic & $\begin{array}{l}\text { Low to moderate }(\mathrm{n}=111) \\
M(S D)\end{array}$ & $\begin{array}{c}\text { Elevated }(\mathrm{n}=17) \\
M(S D)\end{array}$ & $\mathrm{t}(126)$ \\
\hline Age & $54.81(12.875)$ & $52.61(9.382)$ & 1.588 \\
\hline $\begin{array}{l}\text { Satisfaction with } \\
\text { care }\end{array}$ & $67.92(9.044)$ & $60.00(8.937)$ & $3.367 * *$ \\
\hline STAI trait & $37.36(11.966)$ & $56.12(11.407)$ & $-6.054 * *$ \\
\hline Resilience & $80.96(13.461)$ & $60.65(18.517)$ & $4.351 * *$ \\
\hline Total support & $72.95(12.258)$ & $62.47(24.923)$ & 1.703 \\
\hline Family & $24.55(4.552)$ & $22.24(8.635)$ & 1.083 \\
\hline Friend & $23.90(4.189)$ & $18.06(8.927)$ & $2.654 * *$ \\
\hline Significant other & $24.50(6.130)$ & $22.18(8.840)$ & 1.367 \\
\hline \multicolumn{4}{|l|}{ Coping } \\
\hline Emotion & $19.94(5.682)$ & $18.11(2.698)$ & .907 \\
\hline Active & $22.04(6.784)$ & $20.44(7.358)$ & 1.832 \\
\hline Avoidant & $14.61(4.450)$ & $17.17(3.666)$ & $-2.494 *$ \\
\hline Presence of meaning & $27.61(6.262)$ & $20.94(10.968)$ & $3.656 * *$ \\
\hline Search for meaning & $16.70(8.362)$ & $18.33(10.633)$ & -.445 \\
\hline
\end{tabular}

$* p<.05 . * * p<.01$ 
Table 14

Demographic Differences by Depression Level on the HADS-D Scale

Characteristic $\quad$ Low to moderate $(n=109) \quad$ Elevated $(n=17) \quad \chi 2$

Relationship status

Alone

25

5

.390

With another

84

12

Children in home

Yes

32

3

.928

No

77

14

Education

High school or

57

12

2.196

less

College

52

5

Employment

Working

68

10

.037

Not working

41

7

Prior abnormal mammogram

Yes

46

6

.231

No

63

11

Previous breast biopsy

Yes

38

4

.766

No

71

13

Family history breast cancer

Yes

33

5

.063

No

71

11

Unknown

5

1 


\begin{tabular}{lccc}
\hline Personal history of other cancer & & & \\
Yes & 21 & 6 & 2.375 \\
No & 89 & 11 & \\
\hline$* p<.05 . * * p<.01$ & & &
\end{tabular}


Table 15

Multiple Regression Model for STAI State Scale Score

\begin{tabular}{lccc}
\hline Predictor & $B$ & $S E B$ & Significance \\
\hline Trait anxiety & .950 & .842 & $p<.001$ \\
\hline
\end{tabular}


Table 16

Multiple Regression Model for HADS-D Scale Score

\begin{tabular}{lccc}
\hline Predictors & $B$ & $S E B$ & Significance \\
\hline Trait Anxiety & .145 & .440 & $p<.001$ \\
Friend support & -.134 & -.163 & $p=.009$ \\
Presence of meaning & 1.176 & -.295 & $p=.071$ \\
Satisfaction with care & -.051 & -.108 & $p<.001$ \\
\hline
\end{tabular}


Figure 1

Model of UIT (Mishel, 1988)

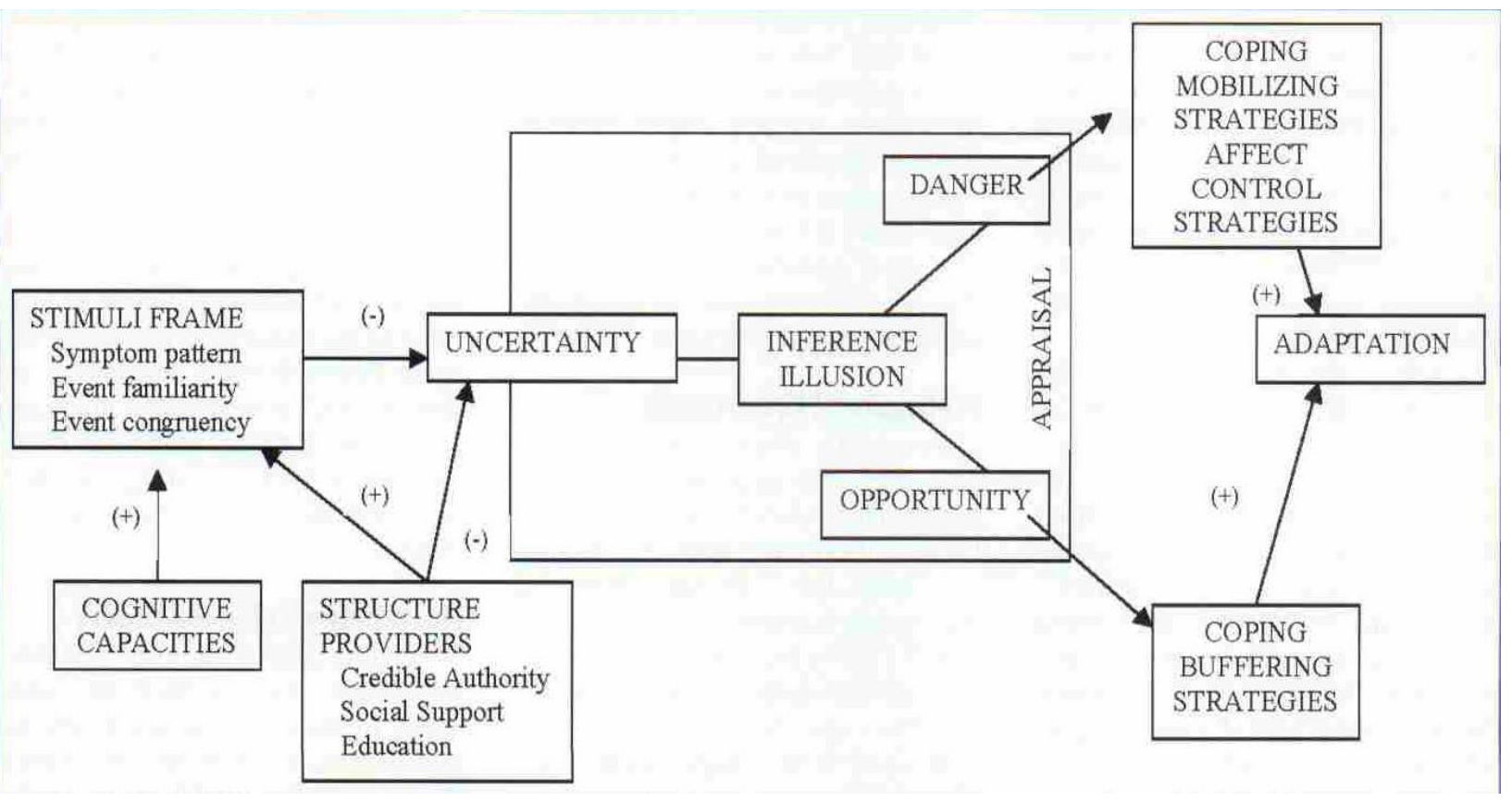

